\title{
Jackiw-Teitelboim supergravity as a double-cut matrix model
}

\author{
Clifford V. Johnson $\odot,{ }^{1, *}$ Felipe Rosso $\odot,{ }^{1, \dagger}$ and Andrew Svesko, ${ }^{2, \ddagger}$ \\ ${ }^{1}$ Department of Physics and Astronomy, University of Southern California, \\ Los Angeles, California 90089-0484, USA \\ ${ }^{2}$ Department of Physics and Astronomy, University College London, \\ Gower Street, London WC1E 6BT, United Kingdom
}

(Received 2 July 2021; accepted 11 September 2021; published 12 October 2021)

\begin{abstract}
We study a Jackiw-Teitelboim (JT) supergravity theory, defined as a Euclidean path integral over orientable supermanifolds with constant negative curvature, which was argued by Stanford and Witten to be captured by a random matrix model in the $\boldsymbol{\beta}=2$ Dyson-Wigner class. We show that the theory is a double-cut matrix model tuned to a critical point where the two cuts coalesce. Our formulation is fully nonperturbative and manifestly stable, providing for explicit unambiguous computation of observables beyond the perturbative recursion relations derivable from loop equations. Our construction shows that this JT supergravity theory may be regarded as a particular combination of certain type $0 \mathrm{~B}$ minimal string theories, and is hence a natural counterpart to another family of JT supergravity theories recently shown to be built from type $0 \mathrm{~A}$ minimal strings. We conjecture that certain other JT supergravities can be similarly defined in terms of double-cut matrix models.
\end{abstract}

DOI: $10.1103 /$ PhysRevD.104.086019

\section{INTRODUCTION}

Jackiw-Teitelboim (JT) gravity [1,2], a theory of 2D dilaton gravity, has emerged as one of the simplest models for studying nontrivial problems in quantum gravity. Describing the low-energy dynamics of a wide class of near-extremal black holes and branes [3-7], JT gravity also features in a precise realization of holographic $\mathrm{AdS}_{2} / \mathrm{CFT}_{1}$, arising as the low-energy gravitational dual to the SachdevYe-Kitaev (SYK) 1D quantum mechanical system [8-13]. It has also been a crucial ingredient in recent work that has yielded new insights into how the black hole information puzzle may be resolved [14-17].

The partition function of the theory $Z(\beta)$ has two natural parameters: the length, $\beta$, of a boundary, which defines the temperature via $\beta=1 / T$ and the quantity $S_{0}$ (which can be thought of as the extremal entropy of the parent black hole), which defines a coupling $\hbar=e^{-S_{0}}$. Surfaces of Euler character $\chi=2(1-g)-n$ (there are $g$ handles and $n$ boundaries) contribute with a factor $\hbar^{-\chi}$ to the path integral. It is vitally important to be able to fully formulate JT gravity as a complete theory of quantum gravity in its own

\footnotetext{
johnson1@usc.edu

felipero@usc.edu

¥a.svesko@ucl.ac.uk
}

Published by the American Physical Society under the terms of the Creative Commons Attribution 4.0 International license. Further distribution of this work must maintain attribution to the author(s) and the published article's title, journal citation, and DOI. Funded by SCOAP ${ }^{3}$. right, not just perturbatively in the parameters, but beyond. A landmark discovery in this regard was the remarkable demonstration by Saad, Shenker and Stanford [18] that JT gravity can be formulated as certain random Hermitian matrix models, in a double scaling [19-22] limit:

$$
Z\left(\beta_{1}, \ldots, \beta_{n}\right) \leftrightarrow\left\langle\operatorname{Tr} e^{-\beta_{1} H} \cdots \operatorname{Tr} e^{-\beta_{n} H}\right\rangle_{c} .
$$

On the left-hand side $Z\left(\beta_{1}, \ldots, \beta_{n}\right)$ is the JT gravity path integral, computed as a Euclidean path integral over (connected) Riemann surfaces with constant negative curvature and $n$ asymptotic boundaries of lengths $\beta_{i}$. On the right-hand side is the connected correlation function of insertions of the operator $\operatorname{Tr} e^{-\beta_{i} H}$, with $\langle\cdots\rangle_{\mathrm{c}}$ implying an ensemble average of the Hermitian matrix $H$. The correspondence was established in Ref. [18] via powerful recursion relations, matching the volumes of moduli spaces of Riemann surfaces [23] contributing to $Z\left(\beta_{1}, \ldots, \beta_{n}\right)$ to a genus expansion of a Hermitian matrix integral $[24,25]$. While Eq. (1) is understood to be order by order in genus $g$ and then summed, a matrix model definition can in principle supply nonperturbative completions that go beyond the sum over genus. Nonperturbative physics is extremely important, especially for studying the low energy regime, and so it is of great interest to fully understand and characterize matrix model formulations, as a means of extracting it. ${ }^{1}$

\footnotetext{
${ }^{1}$ While the Hermitian matrix model definition of Ref. [18] has a problematic nonperturbative definition, a complete nonperturbative completion of ordinary JT gravity that is naturally rooted in matrix models can be found, as shown in Ref. [26].
} 
The correspondence was broadened rather elegantly by Stanford and Witten [27] to include wider classes of JT gravity, incorporating models with fermions and/or time-reversal symmetry. Such models were shown to be classified according to one of the ten types of random matrix ensemble: the three Dyson-Wigner $\boldsymbol{\beta}$ ensembles and the seven Altland-Zirnbauer $(\boldsymbol{\alpha}, \boldsymbol{\beta})$ ensembles $[28,29]$. Many detailed gravity computations [now involving the moduli space of orientable and/or unorientable (super) Riemann surfaces] were shown to be captured by detailed matrix model integrals, establishing that expression (1) still holds (for an appropriate choice of $H$ ). In the case of $\mathcal{N}=1$ JT supergravity, which will be the focus of this paper, the Hermitian matrix is written $H=Q^{2}$, where it is the supercharge $Q$ that is (on general grounds) classified in the tenfold way, depending upon the number, $\mathrm{N}$, of fermions the effective SYK-like "boundary" dual has, and the action of $(-1)^{\mathrm{F}}$ and $\mathrm{T}$, where $F$ and $T$ are fermion number and the time reversal operator, respectively [27,30-36].

Symmetry under $T$ results in the inclusion of unorientable surfaces in the path integral, but this paper will feature JT supergravity theories models that do not preserve $T$. So all that is left is to consider whether $(-1)^{\mathrm{F}}$ is a symmetry or not-i.e., whether $\mathrm{N}$ is even (let us call this case A) or odd (case B). In case A, since the supercharge anticommutes with $(-1)^{\mathrm{F}}$, it can be written [in a basis where $(-1)^{\mathrm{F}}$ is block diagonal] as

$$
Q=\left(\begin{array}{cc}
0 & M \\
M^{\dagger} & 0
\end{array}\right)
$$

where $M$ is a complex matrix, and $M^{\dagger}$ its Hermitian conjugate (see Sec. 2.6.2 in Ref. [27]), and the natural combination the matrix model (1) cares about is $M^{\dagger} M$. In case $\mathrm{B}$, there is no grading due to $(-1)^{\mathrm{F}}$, and so $Q$ is itself just an Hermitian matrix. This case should therefore fall into the $\boldsymbol{\beta}=2$ Dyson-Wigner class.

Recently, it was shown in Refs. [37-39] how to use double-scaled random complex matrix models [40-44] with a potential of the form $V\left(M^{\dagger} M\right)$ to yield a complete perturbative and nonperturbative definition of the case A supergravity above. The formulation was shown to be equivalent to combining together (in a precise sense reviewed later) an infinite family of models known [45] to be equivalent to type $0 \mathrm{~A}$ minimal string theories. ${ }^{2}$ For other studies of JT gravity and supergravity using minimal models, see Refs. [46-50].

In retrospect, the involvement of type $0 \mathrm{~A}$ minimal strings is entirely natural. The world sheet physics of minimal

\footnotetext{
${ }^{2}$ In fact, the same framework includes some T-invariant models too. They all fall into the $(\boldsymbol{\alpha}, \boldsymbol{\beta})=(2 \Gamma+1,2)$ AltlandZirnbauer series, for $\Gamma=0,+\frac{1}{2},-\frac{1}{2}$. Here, integer $\Gamma$ breaks $\mathrm{T}$.
}

string theories are themselves theories of two-dimensional quantum gravity. When fermions are present, there is a spin structure on the surfaces that enter the path integral, and how these are treated defines different types of string theories [51]. Type 0A string theories sum over such spin structures and keep track of them by including a weight factor of $(-1)^{\zeta}$ in the sum, where $\zeta(0$ or $1 \bmod 2)$ is the parity of the spin structure. On the other hand, in the JT classifications above, the boundary $(-1)^{\mathrm{F}}$ directly correlates with the bulk $(-1)^{\zeta}$. Hence with hindsight it is natural that, if minimal strings are going to be relevant at all (and that is thrust upon us by the presence of double-scaled matrix models in both settings) it is indeed type 0A minimal strings that relate to case A above. Indeed, many special properties of the nonperturbative "string equations" that describe type 0A strings, when deployed appropriately (as demonstrated in Ref. [37]) yield many of the remarkable properties of the supergravity models noticed in Ref. [27].

This paper answers the natural question as to whether the remaining T-breaking JT supergravity, case B above, can be given an analogous treatment, yielding a fully computable stable nonperturbative definition of the theory's observables. In light of the previous paragraph there is a natural guess: Since it does not respect $(-1)^{\mathrm{F}}$ and hence results in a sum over bulk geometries that simply ignores $(-1)^{\zeta}$, it ought to have a connection to type $\mathrm{OB}$ minimal strings, which themselves ignore that weight factor by definition. The answer will turn out to be yes, and the $\mathrm{OB}$ connection (anticipated in Ref. [27]) will prove to be correct.

Two more clues help lead to these answers, and they can be found in the leading form of the spectral density for $Q$, denoted here $\rho_{0}(q)$. Since $H=Q^{2}$, this is determined by the Laplace transform of the super-Schwarzian partition function, which yields [31] the density $\rho_{0}^{\mathrm{SJT}}(E)$ of $H$ (with energies $E \geq 0$ ). This gives

$$
\rho_{0}^{\mathrm{SJT}}(E)=\frac{\sqrt{2} \cosh (2 \pi \sqrt{E})}{\pi \hbar \sqrt{E}} \Rightarrow \rho_{0}(q)=\frac{\cosh (2 \pi q)}{\pi \hbar},
$$

since $E=q^{2}$, and after taking into account the Jacobian $d E=2 q d q$ in the Laplace transform integral, where the 2 is absorbed by integrating $q$ over $\mathbb{R}$. (A translation factor of $\gamma^{-1}=1 / \sqrt{2}$ has also been used to go from the gravity density to the matrix model density, as in Ref. [27].)

As noticed in Ref. [27], the Hermitian matrix model for $Q$ therefore has (after double scaling to match gravity) a spectral density that naturally spreads over the entire real line. This is our first clue: The most commonly studied cases of double-scaled Hermitian matrix models usually have support on the half line, resulting from the fact that the double-scaling limit "zooms in" to capture the universal physics to be found at one critical end point of the 

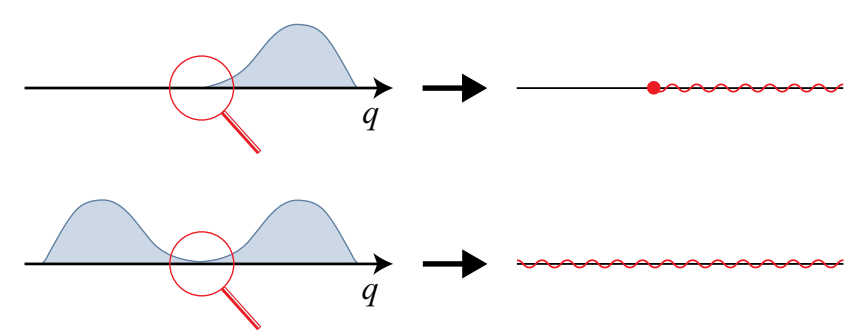

FIG. 1. The double-scaling limit as a "zoom in" to the neighborhood of the end points of spectral densities, resulting in either a semi-infinite cut starting from a single-cut case, or resulting in an infinite cut starting from a double-cut case that has a merger.

(unscaled) spectral density, as shown in the upper part of Fig. 1 (see e.g., Ref. [52] for a review).

The simplest way of arriving at a density that spreads over the whole real line from double scaling is to zoom in on two end points that are colliding with each other, as can happen with critical double-cut matrix models (see the lower part of Fig. 1). The second clue is that $\rho_{0}(q)$ is symmetric on the real line. As will be reviewed later, the string equations that arise from double-scaling symmetric two-cut models (pioneered in Refs. [53-58]) have already been identified in Ref. [45] as capturing type 0B minimal string physics. Moreover, there is a curious universal feature of the string equations that arises in the symmetric sector (the Painlevé II hierarchy) that, as we will show, reproduces certain characteristic features of observables in the JT supergravity. Combining together an infinite family of $0 \mathrm{~B}$ minimal string models in a way precisely analogous to what was done in Refs. [37-39] for the other JT supergravities provides a fully nonperturbative definition of the theory. As an example of what can be computed from it, Fig. 2 displays (blue dots) the full nonperturbative spectral density for $Q$. The black dashed line is the perturbative result of Eq. (3). Other important quantities can be computed in this way, such as the spectral form factor displayed later in Fig. 10.

The rest of this paper is organized as follows. Section II reviews the construction of double-cut Hermitian matrix models and their double-scaling limit. Section III defines the particular model that describes the Dyson-Wigner $\boldsymbol{\beta}=2$ JT supergravity, showing that it reproduces all of the characteristic perturbative results for this model derived in Ref. [27]. Nonperturbative contributions of the matrix model are studied in Sec. IV, where the full spectral density and spectral form factor are obtained by combining analytic and numerical techniques. We finish in Sec. V with some discussion. There are a number of Appendices included that expand some computations, and help keep this work somewhat self-contained without diluting the narrative flow. Appendices A and B are devoted to computing multicorrelators at leading genus and higher genus corrections to the one-point function $\left\langle\operatorname{Tr} e^{-\beta Q^{2}}\right\rangle$, respectively.

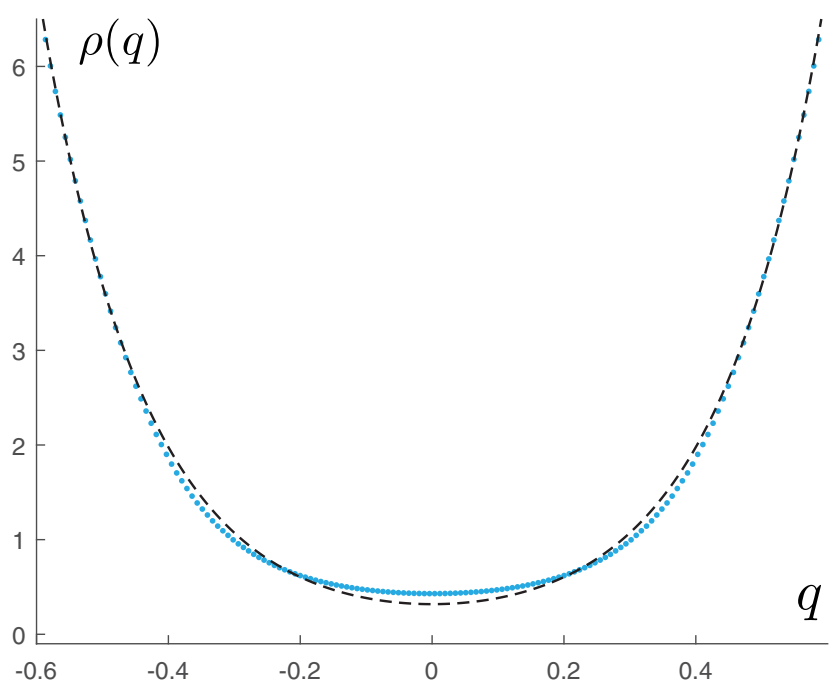

FIG. 2. Full spectral density for the double-cut model relevant for JT supergravity, obtained from summing the two $s= \pm$ contributions shown in Fig. 7. The dashed curve corresponds to the answer to all orders in perturbation theory (3), so that their difference is entirely generated by nonperturbative contributions.

The normalization of eigenfunctions is discussed in Appendix C.

\section{DOUBLE-CUT MATRIX MODELS}

Very soon after the discovery of the double-scaling limit in the context of single-cut Hermitian matrix models in the 1990s [19-22], the study of the double-scaled limit of multicut Hermitian matrix models was initiated in a series of works [55-59], with most of the focus on two-cut models. Closely allied to the latter are the double-scaled unitary matrix models, discovered slightly earlier in Refs. [53,54], which yield a subset of the same physics. ${ }^{3}$ We shall see that in the end, the physics that we need will be accessible from either system, but Hermitian double-cut matrix model language is perhaps the most natural here given the considerations of the previous section. They are built from an $N \times N$ Hermitian matrix $Q,{ }^{4}$ so that the expectation value of a matrix observable $\mathcal{O}$ is

$$
\langle\mathcal{O}\rangle=\frac{1}{\mathcal{Z}} \int d Q \mathcal{O} e^{-N \operatorname{Tr} V(Q)}
$$

where $\mathcal{Z}=\int d Q e^{-N \operatorname{Tr} V(Q)}$ is the matrix partition function. The probability measure is determined by the potential $V(Q)$, that is typically taken to be a polynomial. See

\footnotetext{
${ }^{3}$ Transitions of the kind that interest us, involving mergers of cuts, have an older history from before double-scaling and gravity applications (see e.g., Refs. [60,61]).

${ }^{4}$ Note that the $\operatorname{symbol} N$, the dimension of the square matrix $Q$, should not be confused with the N describing the number of SYK fermions, as used in Sec. I.
} 
Refs. [52,62,63] for useful sources of information about random matrix theory and applications of the doublescaling limit.

A central observable that determines the average distribution of eigenvalues $\lambda_{i} \in \mathbb{R}$ of the matrix $Q$ is the spectral density $\rho(q)$, defined as

$$
\rho(q)=\left\langle\frac{1}{N} \sum_{i=1}^{N} \delta\left(q-\lambda_{i}\right)\right\rangle
$$

Depending on the particular number and location of the minima of $V(Q)$, the spectral density in the large $N$ limit becomes a smooth function of $q$ supported on a finite number of disjoint intervals in $q \in \mathbb{R}$. These intervals ultimately become cuts in the complex $q$-plane when using complex analysis to analyze the large $N$ limit. When $\rho(q)$ is supported on more than one interval, the matrix model is said to be "multicut."

The focus here is on systems that transition between double- and single-cut phases. Precisely at the transition, the corresponding potential $V(Q)$ is said to be "critical," with different critical systems characterized [64] by the rate at which the spectral density vanishes where the two cuts meet at $q=0$, i.e., $\lim _{N \rightarrow \infty} \rho_{m}(q) \sim q^{m}$ with $m \in \mathbb{N}$. For even potentials we have $m=2 k$ with $k \in \mathbb{N}$, and the large $N$ spectral density of a critical system can be written as

$$
\lim _{N \rightarrow \infty} \rho_{2 k}(q)=\frac{b_{k}}{2 \pi}\left(\frac{q}{a}\right)^{2 k} \sqrt{\frac{a^{2}-q^{2}}{a^{2}}},
$$

where $a>0$ and $b_{k}=2^{2 k+1}(k+1) !(k-1) ! / a(2 k-1) !$ is a normalization constant. This spectral density is supported on $q \in(-a, 0) \cup(0, a)$ and arises from a two-cut spectral density transitioning to a single-cut phase. Using standard methods (see e.g., Refs. $[65,66]$ ), the appropriate potential needed to produce this spectral density can be easily worked out to be

$$
V_{2 k}^{\prime}(q)=b_{k} \sum_{n=0}^{k}\left(\begin{array}{c}
1 / 2 \\
n
\end{array}\right)(-1)^{n}\left(\frac{q}{a}\right)^{2(k-n)+1},
$$

which defines the critical model for arbitrary $N$. The even potential $V_{2 k}(q)$ contains two minima symmetrically located in the interval $|q|<a$. Figure 3 is a plot of the potential and spectral density for $k=2$. Note that it is possible to construct models whose spectral density behaves like $\lim _{N \rightarrow \infty} \rho(q) \sim q^{2 k+1}$, but it is more subtle, as these models by themselves are not well defined since the resulting (unscaled) densities are not positive definite. Instead, they can be introduced as perturbations of the even model potentials, as described in Refs. [58,67].

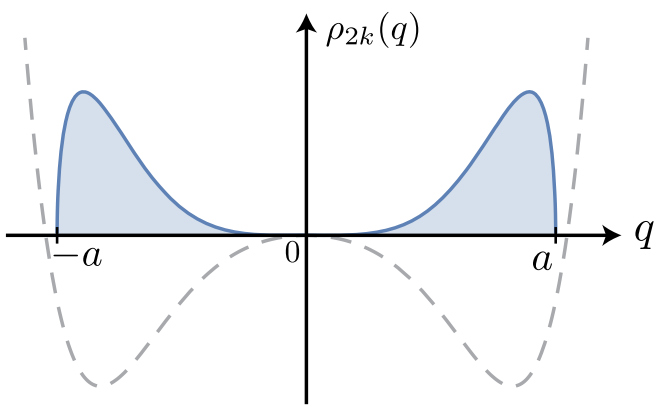

FIG. 3. In blue we plot the singular spectral density (6) with $k=2$, where the spectral density vanishes at the origin were the two cuts meet $\lim _{N \rightarrow \infty} \rho_{2 k}(q) \propto q^{4}$. The dashed curve corresponds to the critical potential (7), with the two minima symmetrically located around $q=0$.

\section{A. The double-scaling limit}

The double-scaling limit involves approaching the critical potential (7) while simultaneously taking the large $N$ limit in a way that captures only certain universal physics associated to the merging of the two cuts. The leading spectral density in the double-scaled model corresponds to the behavior of $\rho(q)$ at $q \sim 0$. For even potentials, this is easily obtained from Eq. (6) by taking $a \rightarrow \infty$, which gives $\rho_{0}(q)=\lim _{N \rightarrow \infty} \rho_{2 k}(q) \sim q^{2 k}$. There will be $1 / N$ (topological) perturbative corrections to this leading large $N$ behavior, and nonperturbative contributions too. There is a powerful formulation (the orthogonal polynomial methods $[68,69])$ that allows for them to be efficiently extracted. These methods are reviewed in e.g., Refs. [52,62,63,65].

A brief summary of the logic is as follows. After writing the matrix model in terms of integrals over the $N$ eigenvalues $\lambda_{n}$, a family of $N$ polynomials $P_{n}(\lambda)=\lambda^{n}+\cdots$ that are orthogonal with respect to the matrix model measure $d \lambda e^{-N V(\lambda)}$ is introduced. A simple argument shows the different polynomials are related according to

$\lambda P_{n}(\lambda)=\sqrt{R_{n+1}} P_{n+1}(\lambda)+S_{n} P_{n}(\lambda)+\sqrt{R_{n}} P_{n-1}(\lambda)$,

where $R_{n}$ and $S_{n}$ depend on the particular theory. The matrix model can be used to derive a family of recursion relations for $R_{n}$ and $S_{n}$, and solving the model is equivalent to finding them. The $P_{n}(\lambda)$ themselves supply a Hilbert space description of the system, on which matrix model observables become operators. This will be very useful shortly. At large $N$, the $R_{n}$ and $S_{n}$ define smooth functions $R(X)$ and $S(X)$ of the variable $X=n / N \in(0,1)$, a smooth coordinate along the spectral density. The scaling limit around the critical behavior at an end point is done by introducing a parameter $b$ which goes to zero as $N \rightarrow \infty$, and the rate at which it does so is set by $\hbar b^{2+1 / m}=1 / N$ (with $m=2 k$ ), determining a scaled topological expansion parameter $\hbar$. The rate at which various quantities in the model approach their critical values at the $X=1$ end point 
is controlled by a power of $b$ e.g., $R(X)=R_{c}+$ $(-1)^{n} b^{1 / m} f(x)+\cdots$, where $x \in \mathbb{R}$ is the scaling part of $X=1-b^{2}(x-\mu)$, and the parameter $\mu$ will be fixed later. A different scaling function $g(x)$ arises from $S(X)$. Inserting all these relations into the recursion relations and taking the limit yields nontrivial coupled ordinary differential equations for $f(x)$ and $g(x)$. These are the socalled "string equations" for the system indexed by $m$.

More generally, the critical potentials (7) can be summed together with coefficients $t_{m}$, and, defining the alternative set of functions $r(x)$ and $\alpha(x)$ by $f(x)=r(x) \cosh (\alpha(x))$ and $g(x)=r(x) \sinh (\alpha(x))$ the differential equations can be conveniently written in the conventions of Ref. [45] as

$$
\begin{aligned}
\sum_{m=1}^{\infty} t_{m} K_{m}+x K_{0} & =0, \\
\sum_{m=1}^{\infty} t_{m} H_{m}+x H_{0}+\eta & =0,
\end{aligned}
$$

where $\eta$ is a parameter of the model, arising as an integration constant (see Ref. [45]) and $\left\{K_{m}, H_{m}\right\}$ are polynomials of $\{r(x), \alpha(x)\}$ and their derivatives determined from the following recursion relations:

$$
\begin{aligned}
& K_{m+1}=\hbar \alpha^{\prime} K_{m}+r H_{m}-\hbar^{2}\left(H_{m}^{\prime} / r\right)^{\prime}, \\
& H_{m+1}^{\prime}=\hbar \alpha^{\prime} H_{m}^{\prime}-r K_{m}^{\prime},
\end{aligned}
$$

where $\left(K_{0}, H_{0}\right)=(r(x), 0)$ and primes are derivatives with respect to $x$ (see Ref. [45] for explicit expressions for the first few values of $m$ ). ${ }^{5}$

The parameter $t_{m}$ controls the double-scaling limit of the critical model with $\rho_{0}(q) \propto q^{m}$, e.g., the doublescaled $2 k$ th model associated to the critical potential in Eq. (7) is obtained by setting all $t_{m}=0$ except for $t_{2 k}=1$. As differential equations, they encode not just the corrections to the leading large $N$ behavior, but nonperturbative information too, as we shall see. They can be derived from the Zakharov-Shabat (ZS) integrable hierarchy [70], in an analog of the manner in which the equations of type $0 \mathrm{~A}$ minimal strings can be derived from the Korteweg-de Vries (KdV) integrable hierarchy. These models were identified as type 0B minimal strings in Ref. [45].

Strictly speaking, we have written here a large family of string equations labeled by any integer $m$. For our purposes, it will be enough to restrict ourselves to the $m$ even models

\footnotetext{
${ }^{5}$ The string equations for the multicut matrix models were first derived in full generality in Refs. [56,58]. We are following the conventions in Ref. [45], slightly changing some of the notation in order to avoid confusion: $\alpha_{\text {here }}=\beta_{\text {there }}, K_{m}^{\text {here }}=R_{m}^{\text {there }}$ and $\eta_{\text {here }}=q_{\text {there }}$. As pointed out in Ref. [45], $q_{\text {there }}$ arises as an integration constant, and counts the amount of R-R flux in a minimal string interpretation.
}

with $\alpha^{\prime}(x)=0 .{ }^{6}$ Using the recursion relations (10) it is straightforward to show that $H_{2 k}=0$, so that the second string equation (9) is automatically satisfied after fixing $\eta=0$. The $K_{2 k}$ are polynomials in $r(x)$ and its derivatives, computed from the following closed recursion relation obtained from Eq. (10):

$$
K_{2 k}=\frac{2 k}{2 k-1}\left[r(x) \int^{x} d \bar{x} r(\bar{x}) K_{2(k-1)}^{\prime}-\frac{1}{4} \hbar^{2} K_{2(k-1)}^{\prime \prime}\right],
$$

where we have rescaled $\hbar \rightarrow \hbar / 2$ as well as $K_{2 k}$ so that they are normalized as $K_{2 k}=r(x)^{2 k+1}+\mathcal{O}\left(\hbar^{2}\right){ }^{7}$ The first few are easily computed and given by

$$
\begin{aligned}
K_{0}= & r(x), \\
K_{2}= & r(x)^{3}-\frac{1}{2} \hbar^{2} r^{\prime \prime}(x), \\
K_{4}= & r(x)^{5}-\frac{5}{6} \hbar^{2} r(x)\left(r(x)^{2}\right)^{\prime \prime}+\frac{1}{6} \hbar^{4} r^{(4)}(x), \\
& \vdots \\
K_{2 k}= & r(x)^{2 k+1}+\cdots+\frac{(-1)^{k} k !(k-1) !}{2(2 k-1) !} \hbar^{2 k} r^{(2 k)}(x),
\end{aligned}
$$

where $r^{(2 k)}(x)$ corresponds to the $2 k x$-derivatives acting on $r(x)$.

Finally, the full string equation for this class of even double-cut models is given by

$$
\sum_{k=1}^{\infty} t_{2 k} K_{2 k}+r(x) x=0 .
$$

Restricting to the even $m$ models and turning off $\alpha(x)$ reduces the two string equations (9), to this simpler single equation. It is in fact the Painlevé II hierarchy of ordinary differential equations (ODEs), related to the modified KdV $(\mathrm{mKdV})$ integrable hierarchy, and was first discovered for double-scaled unitary matrix models $[53,54]$. We shall see that Eq. (13) is enough for describing the JT supergravity theory in which we are interested.

\section{B. Observables}

We shall need to compute certain observables from the function $r(x)$ that solves the string equation (13). The expectation value of any single trace observable can be computed from a certain "macroscopic loop"

\footnotetext{
${ }^{6}$ Note the actual constant value $\alpha(x)=\alpha_{0}$ does not appear in the string equations and is therefore irrelevant.

${ }^{7}$ The rescaling of $K_{2 k}$ can be applied at the level of the string equation by shifting $t_{2 k} \rightarrow t_{2 k} / P_{2 k}(0)$, where $P_{2 k}(z)$ is the Legendre polynomial.
} 
formula derived in Ref. [58]. In our conventions, it is written as [71]

$$
\left\langle\operatorname{Tr}\left(e^{-\beta Q^{2}}\right)\right\rangle=\sum_{s= \pm} \int_{-\infty}^{\mu} d x\left\langle x\left|e^{-\beta \mathcal{H}_{s}}\right| x\right\rangle,
$$

where

$$
\mathcal{H}_{s}=\hat{p}^{2}+\left[r(\hat{x})^{2}-s \hbar r^{\prime}(\hat{x})\right],
$$

with $\hat{p}=-i \hbar \partial_{x}$. The quantum mechanical system spanned by the position eigenstates $|x\rangle$ arises from the continuum limit of the orthogonal polynomial system described in the previous subsection, i.e., $P_{n}(\lambda) \sim|n\rangle$ is promoted to a continuous variable $x[22,72] .{ }^{8}$ The integral comes from the continuum limit, so that a sum over $n$ of orthogonal polynomial quantities, becomes an $X$ integral from 0 to 1 . What remains is the part that survived in the double-scaling limit, which zooms into the neighborhood of the $X=1$ end point defined by $X=1-b^{2}(x-\mu)$, giving $-\infty$ and $\mu$ as the limits on the $x$ integral. The value of $\mu$ will be fixed later by comparison to the supergravity theory. Compared to the single-cut models in Refs. [72,74], there is an additional sum over $s= \pm$ that comes from the fact the orthogonal polynomials have a different behavior for even and odd $n$ [56,71].

The right-hand side is computed in one-dimensional quantum mechanics in the usual way with $\langle p \mid x\rangle=$ $e^{i p x / \hbar} / \sqrt{2 \pi \hbar}$. This provides an extremely concrete formalism that allows us to compute arbitrary single trace observables to all orders in $\hbar$, and nonperturbatively. As explained and shown in Appendix A, analogous formulas can be derived for a higher number of insertions of the matrix operator $\operatorname{Tr} e^{-\beta Q^{2}}$. This will be useful when we compare results of the multicut matrix model to the JT supergravity predictions.

This is all a beautiful counterpart to what occurred for the other JT supergravity model (case A in the Introduction) and its cousins in Refs. [37,39,73]. There, the natural system that arose from double-scaled complex matrix models produced a single Schrödinger Hamiltonian $\mathcal{H}=$ $\hat{p}^{2}+u(\hat{x})$, where $u(x)$ solved a different string equation. All of the techniques applied there can be brought to this system in order to fully solve this JT supergravity. ${ }^{9}$

For a start, we can use Eq. (14) to write a formula for the spectral density $\rho(q)$ using the relation $\left\langle\operatorname{Tr} e^{-\beta Q^{2}}\right\rangle=$ $\int_{-\infty}^{+\infty} d q \rho(q) e^{-\beta q^{2}}$. Defining the wave functions $\psi_{\mathcal{E}, s}(x)$ of the operator $\mathcal{H}_{s}(15)$,

\footnotetext{
${ }^{8}$ See Refs. $[52,63]$ for detailed reviews on how this effective description arises and Refs. [26,37,39,73] for more recent discussions in the JT supergravity context.

${ }^{9}$ In the discussion of Sec. V, there are further comments about the similarities, and crucial differences, between these two systems.
}

$$
\mathcal{H}_{s} \psi_{\mathcal{E}, s}(x)=\mathcal{E} \psi_{\mathcal{E}, s}(x),
$$

with eigenvalue $\mathcal{E}$, a simple calculation yields

$$
\rho(q)=|q| \sum_{s= \pm} \int_{-\infty}^{\mu} d x\left|\psi_{q^{2}, s}(x)\right|^{2} .
$$

Now we are ready to apply all of this technology to the JT supergravity of interest.

\section{PERTURBATIVE PHYSICS}

An important test is to see if the double-cut doublescaled model reproduces the perturbative JT supergravity results obtained in Sec. 5.2.1 of Ref. [27]. Recall that this is a supergravity theory with contributions from orientable supermanifolds of constant negative curvature with both even and odd spin structures weighted equally. As a reminder, we denote as $Z\left(\beta_{1}, \ldots, \beta_{n}\right)$ the partition function with $n$ asymptotic boundaries of renormalized length $\beta_{i}$, which includes all possible (connected) genus surfaces weighted by $\hbar^{2(g-1)+n}$, where $\hbar=e^{-S_{0}}$. These observables were computed in Ref. [27] to all orders in perturbation theory in $\hbar$. They were shown to vanish (see Appendices A and D of Ref. [27]), except for the cases $n=1,2$, which are given by

$$
\frac{Z(\beta)}{\gamma}=\frac{e^{\pi^{2} / \beta}}{\hbar \sqrt{\pi \beta}}, \quad \frac{Z\left(\beta_{1}, \beta_{2}\right)}{\gamma^{2}}=\frac{\sqrt{\beta_{1} \beta_{2}}}{\pi\left(\beta_{1}+\beta_{2}\right)} .
$$

Included with each supergravity observable is an inverse factor of $\gamma=\sqrt{2}$ for each boundary. These are in place in preparation for comparison to matrix model quantities. As explained in Ref. [27], a factor $\gamma$ is needed to convert every matrix model trace involved when comparing to supergravity results.

The spectral density of the supergravity theory, defined from $Z(\beta)=\int_{0}^{\infty} d E \rho_{0}^{\mathrm{SJT}}(E) e^{-\beta E}$, is given on the left of Eq. (3). Meanwhile, the spectral density $\rho_{0}(q)$ of the supercharge via the matrix model is given on the right of Eq. (3). While all of these results are surprisingly simple, we stress that they get corrected by important nonperturbative effects, which we will compute using the methods of this paper in Sec. IV.

\section{A. Matrix model matching}

Section II reviewed a general class of double-scaled matrix models, specified by the coefficients $t_{2 k}$ that determine the string equation (13) satisfied by $r(x)$, as well as by $\mu$ appearing in the computation of observables (14). Fixing to a specific $\left\{t_{2 k}, \mu\right\}$ determines a particular double-scaled model. Matching the leading genus behavior of $\gamma\left\langle\operatorname{Tr} e^{-\beta Q^{2}}\right\rangle$ to the supergravity partition function $Z(\beta)$ in Eq. (18) uniquely fixes these parameters. 
We start by writing a perturbative expansion in $\hbar$ for the function $r(x)$ :

$$
r(x)=r_{0}(x)+\sum_{n=1}^{\infty} r_{n}(x) \hbar^{n}
$$

Inserting this into the string equation (13), the leading contribution $r_{0}(x)$ is determined from the following simple algebraic constraint:

$$
r_{0}(x)\left[\sum_{k=1}^{\infty} t_{2 k} r_{0}(x)^{2 k}+x\right]=0
$$

which admits two possible solutions: Either $r_{0}(x)=0$ or the quantity in parentheses vanishes, giving $r_{0}(x) \neq 0$. To get a nontrivial function $r_{0}(x)$ defined over the whole real line $x \in \mathbb{R}$, we use the piecewise and continuous solution

$$
r_{0}(x): \begin{cases}\sum_{k=1}^{\infty} t_{2 k} r_{0}(x)^{2 k}+x=0, & x \leq 0 \\ r_{0}(x)=0, & x \geq 0 .\end{cases}
$$

From this solution, let us compute the leading genus contribution to $\left\langle\operatorname{Tr} e^{-\beta Q^{2}}\right\rangle$ in Eq. (14), using the following identity:

$$
\left\langle x_{2}\left|e^{-\beta \mathcal{H}_{s}}\right| x_{1}\right\rangle=\frac{e^{-\beta r_{0}\left(x_{2}\right)^{2}-\frac{1}{\beta}\left(\frac{x_{1}-x_{2}}{2 \hbar}\right)^{2}}}{2 \hbar \sqrt{\pi \beta}}+\mathcal{O}(\hbar),
$$

which can be easily proven by inserting a complete set of eigenstates of the momentum operator $\hat{p}$ and solving the resulting $p$ integral. Using this in Eq. (14) we find

$$
\begin{aligned}
\left\langle\operatorname{Tr} e^{-\beta Q^{2}}\right\rangle & =\frac{2}{2 \hbar \sqrt{\pi \beta}} \int_{-\infty}^{\mu} d x e^{-\beta r_{0}(x)^{2}}+\mathcal{O}(\hbar) \\
& \simeq \frac{1}{\hbar \sqrt{\pi \beta}}\left[\mu+\sum_{k=1}^{\infty} t_{2 k} 2 k \int_{0}^{\infty} d r_{0} r_{0}^{2 k-1} e^{-\beta r_{0}^{2}}\right] \\
& =\frac{1}{\hbar \sqrt{\pi \beta}}\left[\mu+\sum_{k=1}^{\infty} \frac{t_{2 k} k !}{\beta^{k}}\right]+\mathcal{O}(\hbar),
\end{aligned}
$$

where in the second equality we changed the integration variable to $r_{0}$ and computed the Jacobian using Eq. (21). Note that to leading order the operator $\mathcal{H}_{s}$ in (15) is independent of $s= \pm$. We have also assumed the boundary condition $r_{0}(-\infty)=+\infty$ that we shall verify shortly.

The leading $\hbar$ behavior in (23) depends on the parameters $\left\{t_{2 k}, \mu\right\}$, and it should match with the supergravity observable $Z(\beta) / \gamma$ in Eq. (18). Clearly, the matrix model result (23) has precisely the right structure, and we find agreement if

$$
\left(t_{2 k}, \mu\right)=\left(\frac{\pi^{2 k}}{k !^{2}}, 1\right)
$$

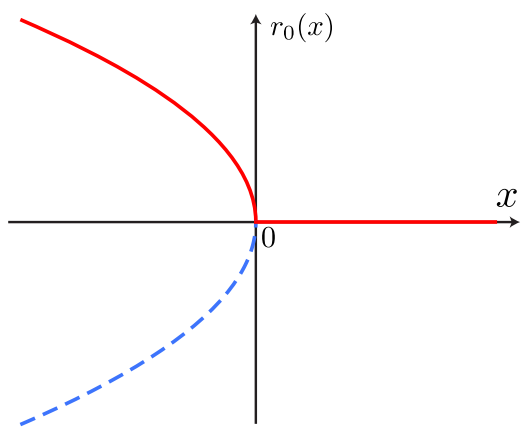

FIG. 4. Plot of the leading solution $r_{0}(x)$ obtained from (21) after fixing $t_{2 k}$ according to (24). In the region $x<0$ there are two solutions to the implicit constraint, indicated in red (solid) and blue (dashed). We chose the red branch, which satisfies the boundary conditions assumed in (23).

This unambiguously defines the multicut double-scaled model. ${ }^{10}$ With this choice, the spectral density $\rho_{0}(q)$ of the matrix model is the correct function given in Eq. (3) and the definition of $r_{0}(x)$ in the $x<0$ region of Eq. (21) can be resummed and written in terms of a modified Bessel function. Specifically, the potential $r_{0}(x)$ satisfies the constraint

$$
I_{0}\left(2 \pi r_{0}\right)-1+x=0
$$

Figure 4 shows $r_{0}(x)$ for $x \in \mathbb{R}$ (red curve), where we see that the assumed boundary condition $r_{0}(-\infty)=+\infty$ is satisfied.

\section{B. Further comparison with JT supergravity}

Fixing the parameters $\left\{t_{2 k}, \mu\right\}$ of the multicut model according to Eq. (24) only ensures the matching between $Z(\beta)$ and $\gamma\left\langle\operatorname{Tr} e^{-\beta Q^{2}}\right\rangle$ to leading order in $\hbar$. Now we must check whether higher trace operators and perturbative corrections reproduce the all-orders supergravity results (18), after using the identification in Eq. (1) where $H=Q^{2}$.

\section{Leading genus multitrace observables}

We start by extending the leading genus analysis in Eq. (23) to multitrace observables. For two and three $\operatorname{Tr} e^{-\beta Q^{2}}$ insertions the computation is reasonably straightforward, starting from the Appendix A general formulas (A1) and (A4). Denoting $G_{0}\left(\beta_{1}, \ldots, \beta_{n}\right)$ as the leading $\hbar$ behavior of the connected expectation value of $n$ insertions of $\operatorname{Tr} e^{-\beta_{i} Q^{2}}$, we derive

\footnotetext{
${ }^{10}$ Note that these agree with the values of the $t_{k}$ parameters found for the complex matrix model definition of the other supergravities discussed in Refs. [37,38].
} 


$$
\begin{aligned}
G_{0}\left(\beta_{1}, \beta_{2}\right) & =2 \frac{\sqrt{\beta_{1} \beta_{2}}}{2 \pi \beta_{T}} e^{-\beta_{T} r_{0}(\mu)^{2}}, \\
G_{0}\left(\beta_{1}, \beta_{2}, \beta_{3}\right) & =2 \frac{\sqrt{\beta_{1} \beta_{2} \beta_{3}}}{2 \pi^{3 / 2} \beta_{T}}\left[\left(\hbar \partial_{x}\right) e^{-\beta_{T} r_{0}(x)^{2}}\right]_{x=\mu},
\end{aligned}
$$

where $\beta_{T}=\sum_{i=1}^{n} \beta_{i}$. Using that $r_{0}(x)$ vanishes for positive $x$ and $\mu>0, G_{0}\left(\beta_{1}, \beta_{2}\right)$ precisely matches $Z\left(\beta_{1}, \beta_{2}\right) / \gamma^{2}$ in Eq. (18) and $G_{0}\left(\beta_{1}, \beta_{2}, \beta_{3}\right)=0$, such that $Z_{0}\left(\beta_{1}, \beta_{2}, \beta_{3}\right)=0$, again in agreement with the supergravity result. ${ }^{11}$

Since the procedure used in Appendix A becomes increasingly tedious when computing higher trace observables, we can instead use a compact formula derived in Refs. [75,76] for the leading genus behavior of single-cut Hermitian matrix models. While single-cut matrix models are in a different universality class, observables are also computed from an almost identical effective quantum mechanical system [52,63]. To leading order, the only difference is that the potential $r_{0}(x)^{2}$ is positive definite and a factor of 2 coming from the summation over $s= \pm$ in (14). Taking this into account, we can apply the general formula of Refs. $[52,75,76]$ to the double-cut case and find

$G_{0}\left(\beta_{1}, \ldots, \beta_{n}\right)=2 \frac{\sqrt{\beta_{1} \cdots \beta_{n}}}{2 \pi^{n / 2} \beta_{T}}\left[\left(\hbar \partial_{x}\right)^{n-2} e^{-\beta_{T} r_{0}(x)^{2}}\right]_{x=\mu}$.

For $n=2,3$ we recover the results in (26). Applying this formula for the $n>3$ cases we find $G_{0}\left(\beta_{1}, \ldots, \beta_{n}\right)$ vanishes for $n \geq 3$, given that $r_{0}(x)$ and all its derivatives vanish at $x=\mu>0$. In summary, we have shown how the doublecut matrix model exactly reproduces all the supergravity results to leading order in genus and arbitrary number of boundaries.

\section{Higher genus corrections}

Next, we compute $\hbar$ corrections to $r_{0}(x)$ in Eq. (19), by solving the string Eq. (13) in perturbation theory. There are two different perturbative expansions, one valid for large positive $x$ and the other for large negative $x$. Since $\mu$ is positive, the relevant one for comparing with supergravity is the large positive $x$ expansion. This is completely analogous to choices present when using the type 0A models, as discussed in Ref. [37]. It is convenient to introduce the parameter $c \in \mathbb{R}_{+}$, and add $\hbar c$ to the right-hand side of the string equation (13), giving

$$
\sum_{k=1}^{\infty} t_{2 k} K_{2 k}+r(x) x=\hbar c
$$

\footnotetext{
${ }^{11}$ Again, as explained in Ref. [27], there is a factor $\gamma=\sqrt{2}$ for every matrix model trace involved when comparing to supergravity results.
}

so that $c=0$ corresponds to the case of interest. Inserting (19) in this differential equation it is straightforward to solve for the first few orders and find

$$
\begin{aligned}
r(x)= & r_{0}(x)+\frac{\hbar c}{x}\left[1+t_{2} \frac{\hbar^{2}\left(1-c^{2}\right)}{x^{3}}+\frac{\hbar^{4}\left(1-c^{2}\right)}{x^{6}}\right. \\
& \left.\times\left(t_{2}^{2}\left(10-3 c^{2}\right)-t_{4}\left(4-c^{2}\right) x\right)+\mathcal{O}\left(\hbar^{6}\right)\right] .
\end{aligned}
$$

Notice that the whole perturbative series vanishes when $c=0$, the case of interest. This is no accident and follows from the observation that $r(x)=0$ is an exact solution to the full string equation when $c=0$. This means the function $r(x)$ for the model relevant to JT supergravity receives no perturbative corrections and is given by $r_{0}(x)$ of Eq. (21) [with $t_{2 k}$ as in (24) and plotted in Fig. 4] to all orders in perturbation theory. We will compute and display the nonperturbative contributions in Sec. IV.

To compute higher order corrections to $\left\langle\operatorname{Tr} e^{-\beta Q^{2}}\right\rangle$ using Eq. (14) essentially involves computing the subleading terms in Eq. (22). Building on some results of Gel'fand and Dikii [77], we derive the following expansion in Appendix B:

$$
\begin{aligned}
& \left\langle x\left|e^{-\beta \mathcal{H}_{s}}\right| x\right\rangle \\
& \simeq \frac{e^{-\beta r_{0}^{2}}}{2 \hbar \sqrt{\pi \beta}}\left\{1+\hbar \beta\left(s r_{0}^{\prime}-2 r_{0} r_{1}\right)\right. \\
& \quad+\frac{\hbar^{2} \beta}{12}\left[\beta^{2}\left[\left(r_{0}^{2}\right)^{\prime}\right]^{2}-12\left(r_{1}^{2}+2 r_{0} r_{2}-s r_{1}^{\prime}\right)\right. \\
& \left.\left.\quad+2 \beta\left(12\left(r_{0} r_{1}\right)^{2}+\left(r_{0}^{\prime}\right)^{2}-2 r_{0} r_{0}^{\prime \prime}-12 s r_{0} r_{1} r_{0}^{\prime}\right)\right]\right\},
\end{aligned}
$$

where the $r_{n}(x)$ are corrections to $r_{0}(x)$ defined in Eq. (19), and " $\simeq$ " means that terms of order $\hbar^{3}$ have been dropped. Integrating this expansion as in (14), we obtain higher $\hbar$ corrections to $\left\langle\operatorname{Tr} e^{-\beta Q^{2}}\right\rangle$. In doing so, there is an important distinction that must be made regarding the leading and subleading terms in Eq. (30).

When integrating (30) only the leading term must be integrated in the whole range $x \in(-\infty, \mu]$ indicated in Eq. (14). All subleading $\hbar$ contributions can only be integrated on the region $x \in[0, \mu]$, where the expansion is meaningful (recall that we did a positive $x$ expansion, not a negative $x$ one, and it is meaningless to have both expansions present in the same expression). But we have already shown that the solution $r_{0}(x)=0$ for $x>0$ (21) receives no perturbative corrections, meaning $r_{n}(x)=0$ for $n \geq 0$. As a result, all $\hbar$ corrections to (30) vanish and we conclude that

$$
\left\langle\operatorname{Tr} e^{-\beta Q^{2}}\right\rangle=\frac{1}{\hbar \sqrt{\pi \beta}} \int_{-\infty}^{\mu} d x e^{-\beta r_{0}(x)^{2}}=\frac{e^{\pi^{2} / \beta}}{\hbar \sqrt{\pi \beta}} .
$$


The important difference with the previous computation in Eq. (23) is that this is an exact result to all orders in perturbation theory, matching with the supergravity result in Eq. (18).

Let us emphasize that the vanishing of higher $\hbar$ corrections to $\left\langle\operatorname{Tr} e^{-\beta Q^{2}}\right\rangle$ does not depend on the special tuning of couplings that define the model, i.e., $\left\{t_{2 k}, \mu\right\}$ in Eq. (24). Instead, it is a consequence of having $r(x)=0$ for $x>0$ to all orders in perturbation theory which, as we can see from Eq. (29), holds for arbitrary $t_{2 k}$.

With this in mind, consider higher $\hbar$ corrections for multiple insertions of the macroscopic loop operator $\operatorname{Tr} e^{-\beta_{i} Q^{2}}$, which via the dictionary (1) will correspond to the JT supergravity partition function with higher genus corrections. For the supergravity of interest, all higher genus corrections vanish. It is interesting to see how this feature emerges from our definition of the double-scaled matrix model. Consider for example the two-point function, $\left\langle\operatorname{Tr}\left(e^{-\beta_{1} Q^{2}}\right) \operatorname{Tr}\left(e^{-\beta_{2} Q^{2}}\right)\right\rangle$. The $\beta_{2}$ macroscopic loop can be expanded in terms of insertions of pointlike operators $\sigma_{2 k}$, the "microscopic loops" [52,78], giving

$$
\left\langle\operatorname{Tr}\left(e^{-\beta_{1} Q^{2}}\right) \operatorname{Tr}\left(e^{-\beta_{2} Q^{2}}\right)\right\rangle=\sum_{k=1}^{\infty} \beta_{2}^{2 k+\frac{1}{2}}\left\langle\operatorname{Tr}\left(e^{-\beta_{1} Q^{2}}\right) \sigma_{2 k}\right\rangle
$$

(in a particular normalization for the $\sigma_{2 k}$ that we will not need to specify here). However, a $\sigma_{2 k}$ insertion is equivalent [22] (fully nonperturbatively) to differentiating the function $r(x)$ with respect to the general coupling $t_{2 k}$, and so in practical terms, the right-hand side of the above is computed by differentiating the explicit $r(x)$ dependence in (14). In the genus expansion therefore, all contributions to the right-hand side contain $t_{2 k}$ derivatives of $r(x)$, which are given by the $\mathrm{mKdV}$ flows:

$$
\frac{\partial r(x)}{\partial t_{2 k}} \propto K_{2 k}^{\prime}[r]
$$

where the $K_{2 k}[r]$, polynomials in $r(x)$ and its derivatives, are characterized in expressions (11) and (12). Since it has been established that $r(x)=0$ at every order in perturbation theory for arbitrary $t_{2 k}$, we see that $\partial r(x) / \partial t_{2 k}$ vanishes, and hence all higher genus contributions to this correlator. This procedure can be iterated to take care of correlators with higher numbers of $\operatorname{Tr} e^{-\beta Q^{2}}$

\footnotetext{
${ }^{12}$ This same feature (but in a different language) was also observed in Sec. 5.2.1 of Ref. [27], where the vanishing of the perturbation series was shown to be independent of the detailed structure of $\rho_{0}(q)$ (3) but relied on $\rho_{0}(q)$ being supported on the whole real line $q \in \mathbb{R}$, leaving no cut end points to source the relevant resolvents. An analogous situation also applies to other JT supergravity theories, as shown in Ref. [37] for the cases $(\boldsymbol{\alpha}, \boldsymbol{\beta})=(\{0,2\}, 2)$.
}

insertions, showing that they also vanish to all orders in perturbation theory.

In summary, in this section we have shown how the double-cut Hermitian matrix model reproduces all of the perturbative results obtained from supergravity computations in Ref. [27].

\section{NONPERTURBATIVE PHYSICS}

While the perturbative expansion of the supergravity observables is surprisingly simple, there can still be nonperturbative contributions that are not captured by the topological expansion. Although some important nonperturbative effects were discussed in Ref. [27], the main methods used there cannot derive generic nonperturbative effects, whether using supergravity or the recursive loop equations technology which defines the matrix model order by order in the genus expansion. As emphasized in Refs. $[26,37,38]$, the advantage of the alternative matrix model techniques used in this paper is that nonperturbative contributions can be explicitly computed because $r(x)$ is defined nonperturbatively by the string equation.

\section{A. A toy model}

Let us start by considering nonperturbative effects in a simple toy model that is relevant for JT supergravity and where everything can be computed analytically. This model is analogous to the Bessel model extensively studied in e.g., Refs. [26,27,37], and is

$$
r(x)=\frac{\hbar c}{x}
$$

with $x>0$. When $c=0, \pm 1$ it is an exact solution to the string equation (28). The eigenfunctions $\psi_{\mathcal{E}, s}(x)$ of the operator $\mathcal{H}_{s}$ (16) can be computed exactly and written in terms of a Bessel function,

$$
\psi_{\mathcal{E}, s}(x)=\frac{1}{\hbar} \sqrt{\frac{x}{2}} J_{\alpha_{s}}(\sqrt{\mathcal{E}} x / \hbar),
$$

where $\alpha_{s}=c+s / 2$ and the other independent solution is discarded since it is not regular at the origin. The normalization constant is fixed using the procedure described in Appendix C. All observables can be constructed from the following kernel:

$$
\begin{aligned}
& L_{s}\left(\mathcal{E}_{1}, \mathcal{E}_{2}\right) \\
& \equiv \int_{0}^{\mu} d x \psi_{\mathcal{E}_{1}, s}(x) \psi_{\mathcal{E}_{2}, s}^{*}(x) \\
& =\hbar^{2}\left[\frac{\psi_{\mathcal{E}_{1}, s}(x) \psi_{\mathcal{E}_{2}, s}^{\prime}(x)-\psi_{\mathcal{E}_{1}, s}^{\prime}(x) \psi_{\mathcal{E}_{2}, s}(x)}{\mathcal{E}_{1}-\mathcal{E}_{2}}\right]_{x=\mu} \\
& =\frac{1}{2}\left(\frac{\mu}{\hbar}\right)^{2}\left[\frac{\zeta_{2} J_{\alpha_{s}}\left(\zeta_{1}\right) J_{\alpha_{s}}^{\prime}\left(\zeta_{2}\right)-\zeta_{1} J_{\alpha_{s}}^{\prime}\left(\zeta_{1}\right) J_{\alpha_{s}}\left(\zeta_{2}\right)}{\zeta_{1}^{2}-\zeta_{2}^{2}}\right],
\end{aligned}
$$


where $\zeta_{i}=\mu \sqrt{\mathcal{E}_{i}} / \hbar$ and the integration region is $x \in[0, \mu]$ since this is the region where $r(x)$ in (34) is defined. The identity in the second line holds for any model and can be derived using that $\psi_{\mathcal{E}, s}(x)$ are eigenfunctions of the operator $\mathcal{H}_{s}{ }^{13}$ The full spectral density $\rho(q)$ computed from (17) is given by

$$
\begin{aligned}
\rho(q) & =|q| \sum_{s= \pm} L_{s}\left(q^{2}, q^{2}\right) \\
& =\sum_{s= \pm} \frac{\mu \zeta}{4 \hbar}\left[J_{\alpha_{s}}(\zeta)^{2}-J_{\alpha_{s}-1}(\zeta) J_{\alpha_{s}+1}(\zeta)\right],
\end{aligned}
$$

where now $\zeta=\mu|q| / \hbar$. This exact result includes both perturbative and nonperturbative contributions.

The case that is relevant for JT supergravity is when $(c, \mu)=(0,1)$, where the spectral density greatly simplifies to

$$
\left.\rho(q)\right|_{(c, \mu)=(0,1)}=\sum_{s= \pm}\left[\frac{1}{2 \pi \hbar}-\frac{s}{4 \pi q} \sin (2 q / \hbar)\right] .
$$

For each value of $s$ we observe there is a perturbative term in $\hbar$ as well as a single nonperturbative correction, given by a fast oscillatory function, see Fig. 5. A remarkable feature of the spectral density is that nonperturbative corrections coming from each $s$ sector precisely cancel each other, so that we are left with the exact result $\rho(q)=1 / \pi \hbar$. Note that this captures the small $q$ behavior of the spectral density $\rho_{0}(q)$ in (3) required to describe the JT supergravity theory.

The cancellation between nonperturbative corrections in this toy model is exclusive to the spectral density and single trace observable. Using the kernel (36) one can easily compute the expectation value of higher trace observables and explicitly show nonperturbative corrections are nonvanishing.

\section{B. The full model}

Let us now consider the full double scaled that is relevant for describing JT supergravity, defined from the values of $\mu$ and $t_{2 k}$ indicated in (24). The analytic computation of nonperturbative contributions is much more challenging in this case, as it is not possible to write a simple solution $\psi_{\mathcal{E}, s}(x)$ as in the toy model (35). However, we can make some progress by computing the eigenfunction $\psi_{\mathcal{E}, s}(x)$ in the usual WKB approximation

$$
\begin{aligned}
\lim _{\hbar \rightarrow 0} \psi_{\mathcal{E}, s}(x)= & \frac{A_{+} \exp \left[\frac{i}{\hbar} \int_{x_{\min }}^{x} d \bar{x} \sqrt{\mathcal{E}-r_{0}(\bar{x})^{2}}\right]}{\left(\mathcal{E}-r_{0}(x)^{2}\right)^{1 / 4}} \\
& +\frac{A_{-} \exp \left[-\frac{i}{\hbar} \int_{x_{\min }}^{x} d \bar{x} \sqrt{\mathcal{E}-r_{0}(\bar{x})^{2}}\right]}{\left(\mathcal{E}-r_{0}(x)^{2}\right)^{1 / 4}},
\end{aligned}
$$

\footnotetext{
${ }^{13}$ In the random matrix theory context, this is sometimes called a Christoffel-Darboux formula, an integral version of an identity satisfied by orthogonal polynomials $[79,80]$.
}

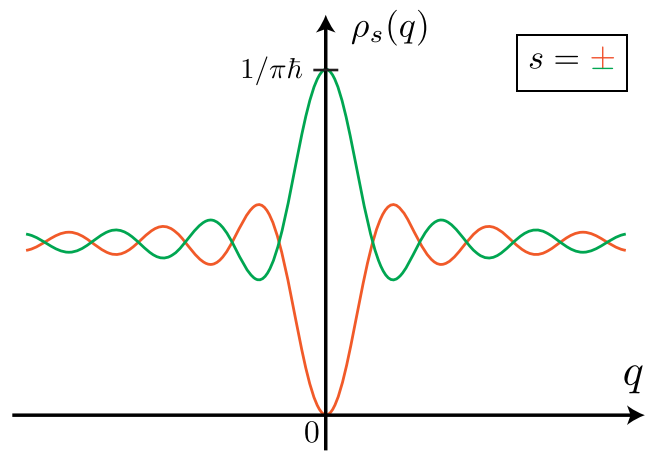

FIG. 5. Plot of the spectral density of the toy model with $(c, \mu)=(0,1)$ in $(38)$, where $\rho_{s}(q)$ corresponds to the $s= \pm$ contribution to the spectral density. While for fixed $s$ we observe nonperturbative corrections, when summing both contribution we get a cancellation which ensures the full spectral density is exactly given by $\rho(q)=1 / \pi \hbar$.

where the parameter $x_{\min }$ is defined from $r_{0}\left(x_{\min }\right)^{2}=\mathcal{E}$, so that this is the solution in the classically allowed region. The challenge is fixing the undetermined constants $A_{ \pm}$. To do so, we note that for any double-scaled model at sufficiently large $x, r(x)$ approaches $r(x)=\hbar c / x$ of the toy model (34). Therefore, for large $x$ the general WKB approximation in (39) should match the classical limit $\hbar \rightarrow 0$ of the eigenfunctions of the toy model (35). In this way, we can fix the constants $A_{ \pm}$and obtain the WKB approximation for an arbitrary model:

$\lim _{\hbar \rightarrow 0} \psi_{\mathcal{E}, s}(x)=\frac{\cos \left[\frac{1}{\hbar} \int_{x_{\min }}^{x} d \bar{x} \sqrt{\mathcal{E}-r_{0}(\bar{x})^{2}}-\frac{\pi}{4}\left(1+2 \alpha_{s}\right)\right]}{\sqrt{\pi \hbar}\left(\mathcal{E}-r_{0}(x)^{2}\right)^{1 / 4}}$.

Setting $x_{\min }=0$ and $r_{0}(x)=0$ we can easily check this expression agrees with the classical limit of (35). For the general case, $x_{\min }$ is a complicated function of $\mathcal{E}$ and $\psi_{\mathcal{E}, s}(x)$ has a different classical limit.

From this we can compute the leading perturbative and nonperturbative contributions to the spectral density $\rho(q)$. Using the expression given in the second line of (36) [which holds for arbitrary $r(x)$ ] as well as the first line in (37) we find

$\lim _{\hbar \rightarrow 0} \rho(q)=\rho_{0}(q)-\sum_{s= \pm} \frac{s}{4 \pi|q|} \sin \left[2 \pi \int^{|q|} d \bar{q} \rho_{0}(\bar{q})-\pi c\right]$,

where we have identified $\rho_{0}(q)$ as the leading perturbative contribution,

$$
\rho_{0}(q)=\frac{|q|}{2 \pi \hbar} \sum_{s= \pm} \int_{x_{\min }}^{\mu} \frac{d x}{\sqrt{q^{2}-r_{0}(x)^{2}}}
$$


and used the following identity:

$$
\int^{|q|} d \bar{q} \rho_{0}(\bar{q})=\frac{1}{\pi \hbar} \int_{x_{\min }}^{\mu} d x \sqrt{q^{2}-r_{0}(x)^{2}} .
$$

The second term in (41) is the interesting one, which gives the leading nonperturbative contribution to $\rho(q){ }^{14}$ The peculiarity is that the contributions from $s= \pm$ precisely cancel each other, so that there is effectively no leading nonperturbative term for the spectral density $\rho(q)$. There can still be subleading contributions, schematically given by

$$
\rho(q)=\rho_{0}(q)+\hbar^{n} \sin (\# / \hbar)+\cdots,
$$

with $n>0$. For the toy model we have seen in (38) that all nonperturbative terms in $\rho(q)$ vanish. It is natural to ask if this is also the case for the matrix model that describes JT supergravity.

To answer this, we need to compute the full eigenvalue spectral density $\rho(q)$, without making any approximation. Since there is no way of performing the calculation analytically, we proceed numerically. The first step is to solve the string Eq. (13) for $r(x)$. To make sense of it as a finite order differential equation, we follow [38] and introduce a truncation by only including contributions from $t_{2 k}$ up to some maximum value $k_{\max }$ :

$$
\sum_{k=1}^{k_{\max }} t_{2 k} K_{2 k}+r(x) x=0,
$$

where $\left(t_{2 k}, \mu\right)$ are given in (24). For high enough values of $k_{\max }$ any artifacts due to the truncation are, at low enough energies, indistinguishable from other numerical errors due to discretization of $r(x)$ or when subsequently solving the spectral problem numerically. From the general structure of $K_{2 k}$ indicated in (12) we see the differential equation for $r(x)$ is of order $2 k_{\max }$. The boundary conditions at large values of $|x|$ are fixed by the leading $\hbar$ solution $r_{0}(x)$ in (21),

$$
\lim _{|x| \rightarrow \infty} \frac{\partial^{n} r(x)}{\partial x}=\lim _{|x| \rightarrow \infty} \frac{\partial^{n} r_{0}(x)}{\partial x},
$$

with $n=0,1, \ldots, k_{\max }-1$. Similar truncation procedures have been successfully applied to other double-scaled matrix models in relation to JT gravity and supergravity [39,73].

In the diagram of Fig. 6, the blue solid line corresponds to the numerical solution for $r(x)$ with $\hbar=1$ and a truncation with $k_{\max }=6$. Working with higher truncations

\footnotetext{
${ }^{14} \mathrm{~A}$ different calculation in Appendix E of [27] gives similar (and in some sense equivalent) result for other random matrix ensembles.
}

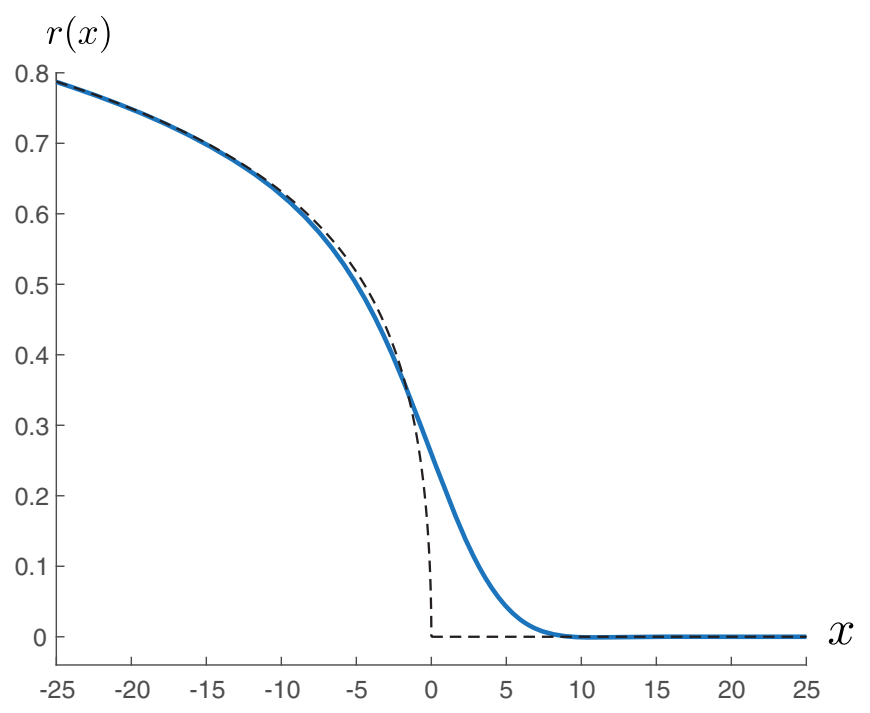

FIG. 6. The solid blue curve corresponds to the full nonperturbative numerical solution $r(x)$ to the string Eq. (13) in the $k_{\max }=6$ truncation with $\hbar=1$. The dashed red curve is the leading genus solution $r_{0}(x)$ in (21).

does not generate any substantial difference, at least for the numerical precision required for the computations here. The dashed line corresponds to $r_{0}(x)$ in (21), that is actually the exact solution to all orders in perturbation theory. The substantial difference between $r(x)$ and $r_{0}(x)$ for small values of $x$ is entirely due to nonperturbative effects.

Using this solution we can compute $r(x)^{2}-s \hbar r^{\prime}(x)$, build the operator $\mathcal{H}_{s}$ and numerically compute the corresponding eigenfunctions $\psi_{\mathcal{E}, s}(x)$, similarly as previously done in $[26,37,38,73]$. Although the eigenfunctions are not normalizable, the normalization constant is fixed from the procedure explained in Appendix C. In this way, we can use (17) and compute the full spectral density $\rho(q)$ that is relevant for JT supergravity, including all perturbative and nonperturbative contributions. For each $s= \pm$ sector we plot the spectral density in Fig. 7. Comparing with the perturbative answer shown in the dashed line, we observe nonperturbative corrections are present and dominate the spectral density for each value of $s= \pm$ at low values of $q$. The actual spectral density of the matrix model $\rho(q)$ is obtained from summing the two $s= \pm$ contributions, shown in the plot of Fig. 2. Comparing with the perturbative answer shown in the dashed line, we observe there are indeed small subleading nonperturbative corrections generated by contributions of the schematic form given in (44).

Since the difference between the perturbative and full spectral density in Fig. 2 is quite small, we want to make sure it is not generated by numerical uncertainties but real physics. To do that, we can take smaller values of $\hbar$, where we expect nonperturbative effects to disappear. As noticed in [38], instead of taking $\hbar$ small, it is numerically more convenient to increase $\mu$. This has the same effect, as larger 


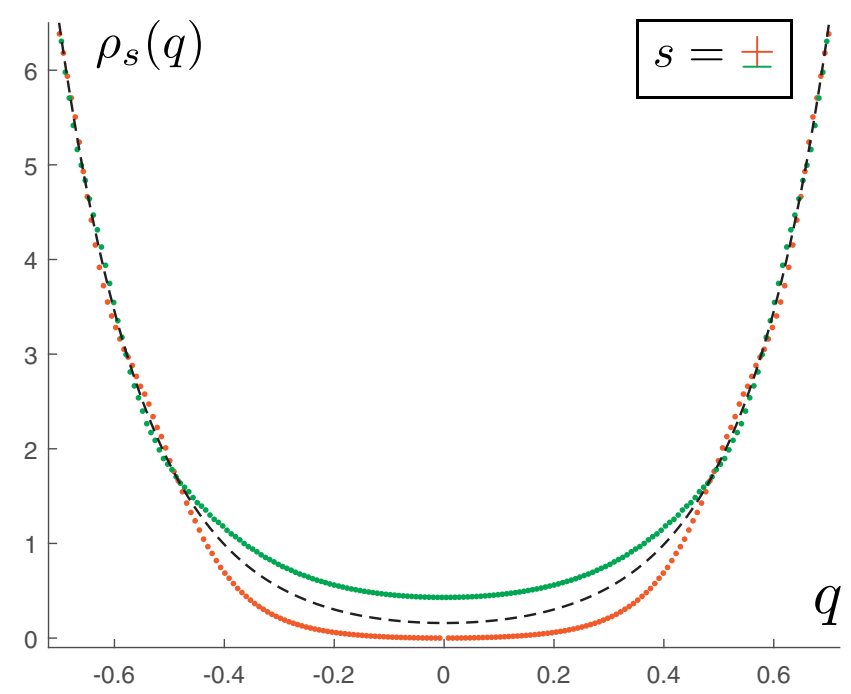

FIG. 7. Plot of each spin contribution $s= \pm$ to the spectral density for $\mu=1$, with the dashed line corresponding to the perturbative answer $\rho_{0}(q) / 2$.

values of $\mu$ means the $x$ integral in (17) gets a larger contribution from the classical region of the potential in $\mathcal{H}_{s}$, suppressing the quantum effects. In Fig. 8 we plot each spin contribution $s= \pm$ to the spectral density when $\mu=10$, instead of $\mu=1$ as in (24). The gray curves in that diagram correspond to the WKB approximation in (41), where we find good agreement. ${ }^{15}$

The full spectral density at $\mu=10$ is obtained by summing these contributions. Instead of doing that it is convenient to isolate the nonperturbative effects by plotting $\rho(q)-\rho_{0}(q)$, shown in Fig. 9 for $\mu=10$ (red) and $\mu=1$ (blue). As expected, we observe the nonperturbative corrections that are present for $\mu=1$ are suppressed as $\mu$ increases and the answer is dominated by the WKB approximation (41). This provides good evidence that the nonperturbative effects seen for $\mu=1$ in Fig. 2 are real physics and not artifacts of the numerics.

Having the nonperturbatively computed eigenfunctions $\psi_{\mathcal{E}, s}(x)$ allows for the computation of many other observables of interest, including all perturbative and nonperturbative corrections. A quantity of particular interest is the spectral form factor, defined as the two-point correlator involving two asymptotic boundaries, written as a sum of disconnected and connected pieces:

$$
\begin{aligned}
\langle Z(\beta+i t) Z(\beta-i t)\rangle \equiv & Z(\beta+i t) Z(\beta-i t) \\
& +Z(\beta+i t, \beta-i t),
\end{aligned}
$$

where the two boundary lengths $\beta_{1}$ and $\beta_{2}$ have been analytically continued to $\beta+i t$ and $\beta-i$. This quantity is

\footnotetext{
${ }^{15} \mathrm{~A}$ similar matching for a different class of double-scaled matrix models that are relevant for other JT supergravity theories was performed in Ref. [38].
}

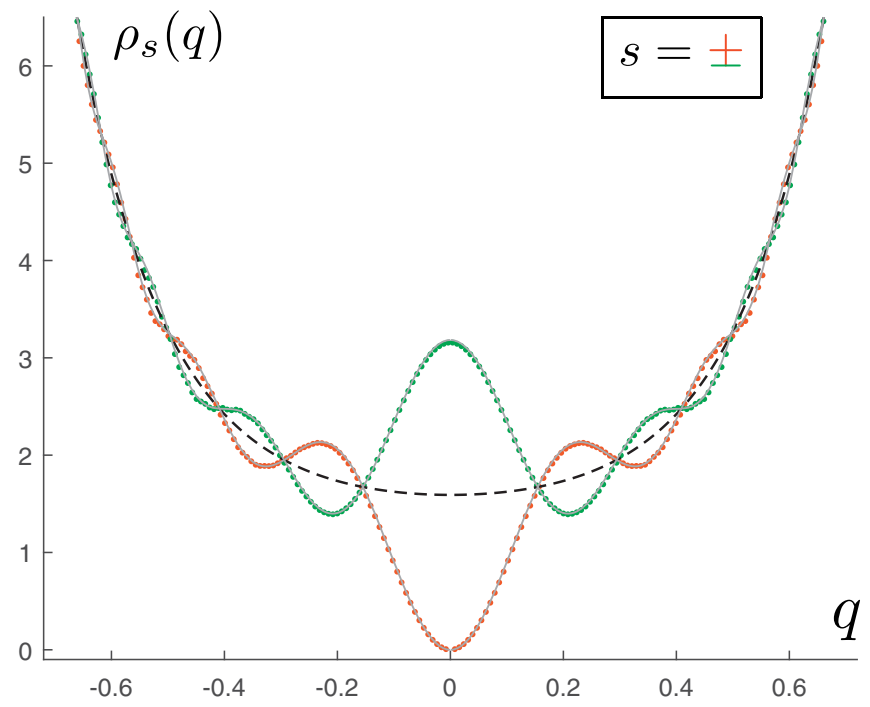

FIG. 8. Plot of each spin contribution $s= \pm$ to the spectral density for $\mu=10$. In this regime, there is good matching with the WKB expression in (41), shown as the gray curves.

useful for diagnosing certain universal aspects of quantum chaotic behavior [81-83]. Random matrix theory predicts the spectral form factor to exhibit "dip," "ramp" and "plateau" behavior, describing the interaction of eigenvalues. In the context of the JT gravity/SYK correspondence, these features of SYK describe aspects of the information scrambling properties of black holes [81].

Using the identification in Eq. (1), the spectral form factor can be computed from the matrix model side by inserting two operators $\operatorname{Tr} e^{-\beta Q^{2}}$ and performing the analytic continuation. For other JT supergravity theories this has been recently computed in Refs. [38,39]

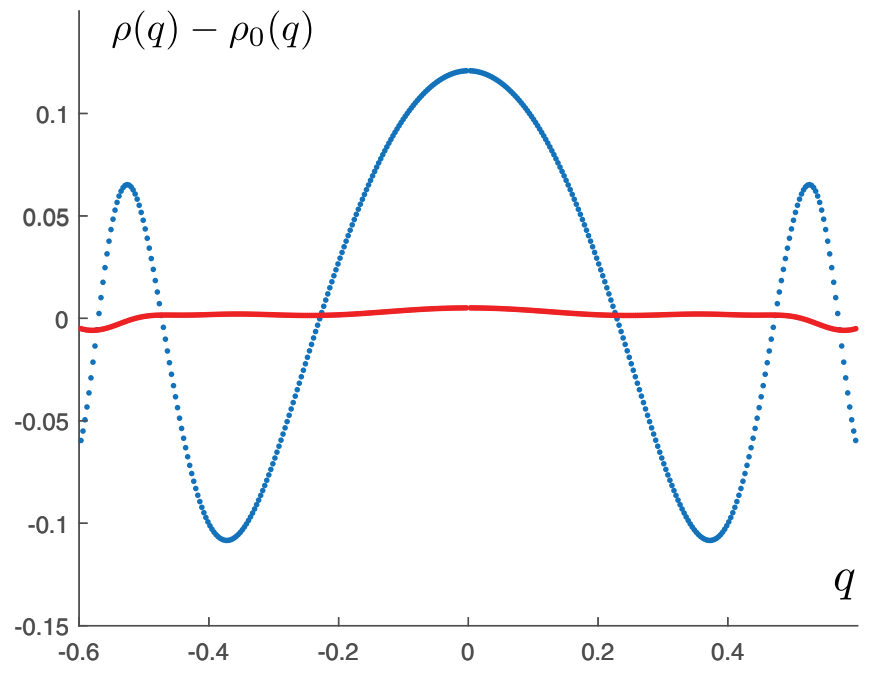

FIG. 9. Difference between the full spectral density $\rho(q)$ and the perturbative result $\rho_{0}(q)$ for $\mu=1$ (blue) and $\mu=10$ (red). We explicitly observe how the nonperturbative corrections are suppressed as the value of $\mu$ increases. 


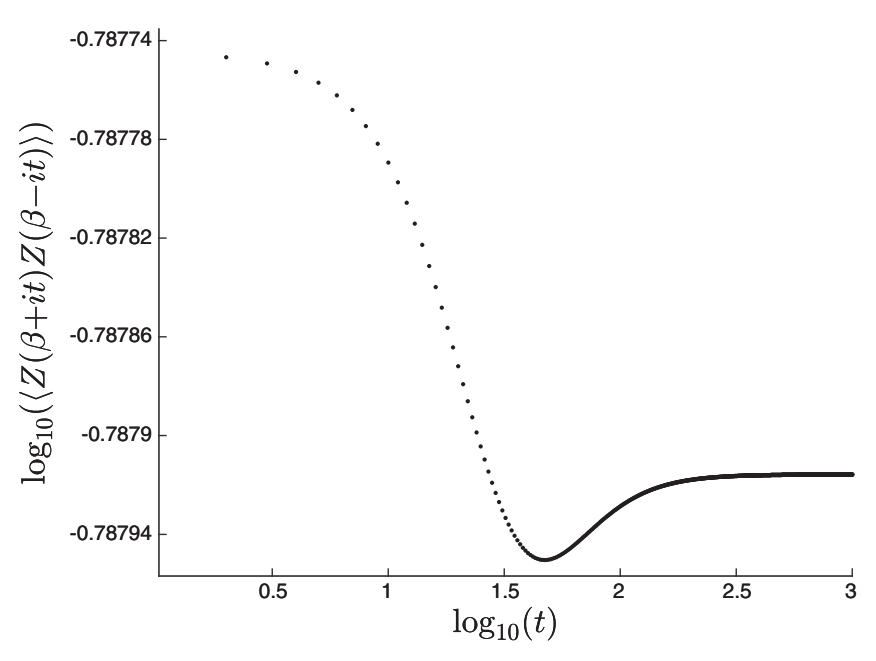

FIG. 10. Full nonperturbative spectral form factor vs time $t$, computed from the matrix model side with $\beta=50$ and $\hbar=1$, using (14), (A1), and the methods described in Refs. [38,39].

[see Eq. (26) of Ref. [38]). Using the eigenfunctions $\psi_{\mathcal{E}, s}(x)$ relevant to this case, we compute an example and obtain the result shown in Fig. 10, showing the expected features. As in the previous work, it is observed that nonperturbative effects again play a crucial role in developing the plateau, and (since $\hbar=1$ in the figure) rapidly take over and hide the usually linear part of the ramp. For brevity, we have omitted showing plots of the individual disconnected portions of the spectral form factor, but there is a great deal of similarity to the $(0,2)$ case studied in Ref. [38], and so we refer the reader there for results and extended discussion. In particular, the initial slope of the falloff of the disconnected piece is unity, as it should be for a JT supergravity [this follows from the leading small $\beta$ dependence of $Z(\beta)$ ]. Also, following from the fact that the spectral density $\rho(E)$ diverges (even after nonperturbative corrections) as $E \rightarrow 0$, the connected piece takes much longer to saturate to the plateau value, just as happened for the $(0,2)$ model.

\section{CLOSING REMARKS}

We have shown how a particular JT supergravity theory-one which sums only over orientable surfaces and equally weights spin structures in the Euclidean path integral-is given a complete nonperturbative description by a double-cut Hermitian matrix model (i.e., a $\boldsymbol{\beta}=2$ Dyson-Wigner ensemble). While generally the double-cut matrix model is characterized by two string equations associated to the ZS integrable hierarchy, here the two string equations collapse to a single string equation, the Painlevé II/mKdV integrable hierarchy, also known from unitary matrix models. These equations are known to describe type $0 \mathrm{~B}$ minimal strings [45], and we showed a precise combination of such minimal models reproduces the JT supergravity. This is completely analogous to the work of Refs. [37-39], which produced similar results for the Altland-Zirnbauer $(\boldsymbol{\alpha}, \boldsymbol{\beta})=(\{0,1,2\}, 2)$ JT supergravities, showing how to construct them from type $0 \mathrm{~A}$ minimal strings.

It was observed long ago in Ref. [42] that there is a nonperturbative map between the string equations of the complex matrix models (type 0A minimal strings) and those of the unitary matrix models (the type 0B minimal strings). It is in fact the Miura transformation [84,85] that links the parent $\mathrm{KdV}$ and $\mathrm{mKdV}$ hierarchies: $u(x)=r(x)^{2}+\hbar r^{\prime}(x)$, where the $v(x)$ of Ref. [42] is $r(x)$ here. (This is different from the map for the $k=1$ case noticed in Ref. [86], or the connection more recently discussed for unscaled models in Ref. [87].) In this sense, the $(\boldsymbol{\alpha}, \boldsymbol{\beta}=2)$ JT supergravities, nonperturbatively defined as complex matrix models in Refs. [37,38], can straightforwardly be cast in terms of unitary matrix model language. ${ }^{16}$ This does not mean that such a recasting captures the Dyson-Wigner $\boldsymbol{\beta}=2$ JT supergravity discussed in this paper however. The key new ingredient, arising from using the loop operator in Eq. (14), is that for the $\boldsymbol{\beta}=2$ case, the physics comes from summing the results of the two separate Schrödinger systems with potentials $r(x)^{2} \pm \hbar r^{\prime}(x)$, and not just a single copy of one or (equivalently) the other, which is appropriate for the other family of JT supergravities. It is a subtle, but crucial, difference.

In a sense, from a nonperturbative (geometryindependent) high-altitude perspective, the fundamental variable is really $r(x)$, and the two different (inequivalent) uses of it to define potentials amounts to the two (inequivalent) JT supergravity families. This perspective deserves further exploration, since this simple difference in choice changes major features of the theory, such as whether it has nonorientable geometries (preserves $\mathrm{T}$ ), respects $(-1)^{\mathrm{F}}$, and so on.

It is worth noting that there is a language in which the two different uses of $r(x)$ are both natural. The integrable hierarchies in question have an underlying $\operatorname{sl}(2, \mathbb{C})$ structure [89-94] from which naturally arises two " $\tau$-functions," $\tau_{0}$ and $\tau_{1}$. In terms of these, define $u_{0,1}=2 \hbar^{2} \partial_{x}^{2} \ln \tau_{0,1}$ where either choice $u_{0,1}=v^{2} \pm \hbar v^{\prime}$ gives a KdV-type function $u(x)$, and recall that $v(x)$ is the $r(x)$ of this paper. Their difference gives $v=\hbar \partial_{x} \ln \left(\tau_{0} / \tau_{1}\right)$ while their sum gives $v^{2}=\hbar^{2} \partial_{x}^{2} \ln \left(\tau_{0} \tau_{1}\right)$. Perhaps this $\tau$-function language can be aligned with the supergravity symmetries $T$ and $(-1)^{\mathrm{F}}$.

There are a number of other interesting potential avenues for future work. For example, as discussed in Ref. [27], there are several other JT supergravity theories that are T invariant (so they include nonorientable surfaces in the Euclidean path integral) and have not yet been studied nonperturbatively. For example, two of them are classified

\footnotetext{
${ }^{16}$ In fact, this was done later in Ref. [88].
} 
as double scaled $\boldsymbol{\beta}=1,4$ Dyson-Wigner ensembles [95-97], and ought to share many of the qualitative features discussed in this paper. In particular, the leading spectral density $\rho_{0}$ for these models has a branch cut comprising the entire real line. It is natural to conjecture (and we do) that these SJT models may be similarly described by double-cut matrix models. It is a natural guess that the string equations for those systems will define a function analogous to $r(x)$ that has a regime where it vanishes to all orders in perturbation theory. The details will be different however, with important nonperturbative differences, since the underlying orthogonal polynomials (and hence the string equations) arising from double scaling will be different.

As already noted, the larger system of equations describing double-cut Hermitian matrix models are from the Zakharov-Shabat hierarchy, where the function $\alpha(x)$ and the parameter $\eta$ (see Sec. II A) are turned on. In our matching to the JT supergravity we restricted to the symmetric sector and turned those off. However, Ref. [45] gave a type $\mathrm{OB}$ minimal string interpretation to some of the more general solutions in terms of symmetry breaking R-R fluxes. It would be interesting to explore if those can be understood in terms of the JT supergravity.

Notably, the KdV, $\mathrm{mKdV}$, and ZS hierarchies are all naturally embedded in a larger hierarchy of differential equations called the dispersive water wave hierarchy (see Ref. [98] and references therein). It was noticed [99,100] that it is possible to derive string-equation-like ODEs from that larger system that have many properties of minimal string theories, suggesting a large interconnected web of minimal string theories of which type $0 \mathrm{~A}$ and type $0 \mathrm{~B}$ are merely a small part. It is tantalizing to imagine that (by using the procedures used here and elsewhere for building JT gravity models out of minimal strings) an interconnected web of JT gravity and supergravity theories can similarly be defined.

Lastly, it would be worthwhile to use complex matrix models and double-cut Hermitian matrix models to describe nontrivial deformations of JT supergravity analogous to those described in Refs. [101,102], and also to JT gravity in de Sitter space $[103,104]$. Nonperturbative insights into such deformations would be useful. Progress has already been made for deformed JT gravity [73], and is currently under way for JT supergravity [71].

\section{ACKNOWLEDGMENTS}

C. V. J. and F. R. are partially supported by the DOE Grant No. DE-SC0011687. A.S. is supported by the Simons Foundation through It from Qubit: Simons collaboration on Quantum Fields, Gravity, and Information.

\section{APPENDIX A: HIGHER TRACE OPERATORS}

In this Appendix we show how to compute the expectation value of higher trace operators in the double-scaled multicut model. Starting from the single trace formula in Eq. (14) one way of deriving the analogous higher trace formulas is to use the free fermion formalism [52,72]. While this formalism was originally developed for singlecut matrix models, the effective quantum mechanical system that arises when computing observables is basically the same after some simple redefinitions.

\section{Double trace operators}

Starting from Eq. (14), we obtain the following formula for two insertions of $\operatorname{Tr} e^{-\beta Q^{2}}$ [71]:

$$
\left\langle\operatorname{Tr}\left(e^{-\beta_{1} Q^{2}}\right) \operatorname{Tr}\left(e^{-\beta_{2} Q^{2}}\right)\right\rangle_{c}=\sum_{s= \pm} \int_{-\infty}^{\mu} d x_{1} \int_{\mu}^{+\infty} d x_{2}\left\langle x_{1}\left|e^{-\beta_{1} \mathcal{H}_{s}}\right| x_{2}\right\rangle\left\langle x_{2}\left|e^{-\beta_{2} \mathcal{H}_{s}}\right| x_{1}\right\rangle
$$

Using Eq. (22), it is quite simple to evaluate its leading $\hbar$ behavior and find

$$
\left\langle\operatorname{Tr}\left(e^{-\beta_{1} Q^{2}}\right) \operatorname{Tr}\left(e^{-\beta_{2} Q^{2}}\right)\right\rangle_{c}=\frac{1}{2 \pi \hbar^{2} \sqrt{\beta_{1} \beta_{2}}} \int_{-\infty}^{\mu} d x_{1} \int_{\mu}^{+\infty} d x_{2} e^{-\beta_{1} r_{0}\left(x_{1}\right)^{2}-\beta_{2} r_{0}\left(x_{2}\right)^{2}-\frac{\beta_{1}+\beta_{2}}{\beta_{1} \beta_{2}}\left(\frac{x_{1}-x_{2}}{2 \hbar}\right)^{2}}+\mathcal{O}(1) .
$$

Changing the integration variables to $x_{1}=\hbar\left(w_{1}-w_{2}\right)+\mu$ and $x_{2}=\hbar\left(w_{1}+w_{2}\right)+\mu$ the integrals decouple after we expand the exponentials $e^{-\beta_{i} r_{0}\left(x_{i}\right)^{2}} \simeq e^{-\beta_{i} r_{0}(\mu)^{2}}$ to leading order in $\hbar$. The remaining $\left(w_{1}, w_{2}\right)$ integrals can be easily solved and we find

$$
\left\langle\operatorname{Tr}\left(e^{-\beta_{1} Q^{2}}\right) \operatorname{Tr}\left(e^{-\beta_{2} Q^{2}}\right)\right\rangle_{c}=2 \frac{\sqrt{\beta_{1} \beta_{2}}}{2 \pi \beta_{T}} e^{-\beta_{T} r_{0}(\mu)^{2}}+\mathcal{O}\left(\hbar^{2}\right),
$$

where we have defined $\beta_{T}=\sum_{i=1}^{n} \beta_{i}$, in this case with $n=2$. Using $r_{0}(\mu)=r_{0}(1)=0$, the leading behavior reproduces the supergravity result in Eq. (18). 


\section{Triple trace Operators}

Let us now consider three insertions of the matrix operator $\operatorname{Tr} e^{-\beta Q^{2}}$. The general formula in this case can be obtained from the neat general expression derived in Ref. [105], and written in Eq. (3.28) of Ref. [106]. In our conventions, it is given by

$$
\begin{aligned}
\left\langle\operatorname{Tr}\left(e^{-\beta_{1} Q^{2}}\right) \operatorname{Tr}\left(e^{-\beta_{2} Q^{2}}\right) \operatorname{Tr}\left(e^{-\beta_{3} Q^{2}}\right)\right\rangle_{c}= & \sum_{s= \pm} \int_{-\infty}^{\mu} d x_{1} \int_{\mu}^{+\infty} d x_{2}\left\langle x_{1}\left|e^{-\beta_{1} \mathcal{H}_{s}}\right| x_{2}\right\rangle \\
& \times\left[\int_{\mu}^{+\infty} d x_{3}\left\langle x_{2}\left|e^{-\beta_{2} \mathcal{H}_{s}}\right| x_{3}\right\rangle\left\langle x_{3}\left|e^{-\beta_{3} \mathcal{H}_{s}}\right| x_{1}\right\rangle-\int_{-\infty}^{\mu} d x_{3}\left\langle x_{2}\left|e^{-\beta_{3} \mathcal{H}_{s}}\right| x_{3}\right\rangle\left\langle x_{3}\left|e^{-\beta_{2} \mathcal{H}_{s}}\right| x_{1}\right\rangle\right] .
\end{aligned}
$$

To evaluate its leading $\hbar$ behavior, which we denote as $G_{0}\left(\beta_{1}, \beta_{2}, \beta_{3}\right)$, we set without loss of generality $\beta_{2}=\beta_{3}=\beta$, as the general answer is then obtained by requiring its symmetric under arbitrary exchanges of $\beta_{i} \leftrightarrow \beta_{j}$. Using (22) we find

$$
\begin{aligned}
G_{0}\left(\beta_{1}, \beta, \beta\right)= & 2 \frac{\sqrt{\beta_{1} \beta^{2}}}{2 \pi^{3 / 2}} \int_{-\infty}^{+\infty} \frac{d x_{3}}{4 \beta_{1} \beta^{2} \hbar^{3}} \operatorname{sign}\left(x_{3}-\mu\right) \int_{-\infty}^{\mu} d x_{1}\left(\int_{\mu}^{+\infty} d x_{2} e^{-\beta_{1} r_{0}\left(x_{1}\right)^{2}-\frac{1}{\beta_{1}}\left(\frac{x_{1}-x_{2}}{2 \hbar}\right)^{2}}\right. \\
& \left.\times e^{-\beta r_{0}\left(x_{2}\right)^{2}-\frac{1}{\beta}\left(\frac{x_{2}-x_{3}}{2 \hbar}\right)^{2}} e^{-\beta r_{0}\left(x_{3}\right)^{2}-\frac{1}{\beta}\left(\frac{x_{1}-x_{3}}{2 \hbar}\right)^{2}}\right) .
\end{aligned}
$$

Changing the integration variables to $x_{i}=\hbar \bar{x}_{i}+\mu$ we can use the following expansion in $\hbar$ :

$$
e^{-\beta_{i} r_{0}\left(x_{i}\right)^{2}}=e^{-\beta_{i} r_{0}(\mu)^{2}}\left[1-\beta_{i}\left[\left(\hbar \partial_{x}\right) r_{0}(x)^{2}\right]_{x=\mu} \bar{x}_{i}+\mathcal{O}\left(\hbar^{3}\right)\right] .
$$

The first term contributes to (A5) as an integral over the whole real line in $\bar{x}_{3}$ of the following function:

$$
F\left(\bar{x}_{3}\right) \equiv \operatorname{sign}\left(\bar{x}_{3}\right) \int_{-\infty}^{0} d \bar{x}_{1} \int_{0}^{+\infty} d \bar{x}_{2} e^{-\frac{\left(\bar{x}_{1}-\bar{x}_{2}\right)^{2}}{4 \beta_{1}}-\frac{\left(\bar{x}_{2}-\bar{x}_{3}\right)^{2}}{4 \beta}-\frac{\left.\bar{x}_{1}-\bar{x}_{3}\right)^{2}}{4 \beta}}
$$

It is straightforward to show this is an odd function $F\left(\bar{x}_{3}\right)=-F\left(-\bar{x}_{3}\right)$, so that it vanishes when integrated over the whole real line. This means the leading contribution is given by the second term in (A6), so that (A5) can be written as

$$
G_{0}\left(\beta_{1}, \beta, \beta\right)=2 \frac{\sqrt{\beta_{1} \beta^{2}}}{2 \pi^{3 / 2}\left(\beta_{1}+2 \beta\right)}\left[\left(\hbar \partial_{x}\right) e^{-\left(\beta_{1}+2 \beta\right) r_{0}(x)^{2}}\right]_{x=\mu}\left\{\frac{1}{4 \beta_{1} \beta^{2}} \sum_{i=1}^{3} \beta_{i} I_{i}\right\},
$$

where we have defined

$$
I_{i}=\int_{-\infty}^{+\infty} d \bar{x}_{3} \operatorname{sign}\left(\bar{x}_{3}\right) \int_{-\infty}^{0} d \bar{x}_{1} \int_{0}^{+\infty} d \bar{x}_{2} \bar{x}_{i} e^{-\frac{\left(\bar{x}_{1}-\bar{x}_{2}\right)^{2}}{4 \beta_{1}}-\frac{\left(\bar{x}_{2}-\bar{x}_{3}\right)^{2}}{4 \beta}-\frac{\left(\bar{x}_{1}-\bar{x}_{3}\right)^{2}}{4 \beta}}
$$

To evaluate these triple integrals, we first apply a change of coordinates that decouples the exponents,

$$
\begin{aligned}
& 3 \bar{x}_{1}=\sqrt{2 \beta \beta_{1}}\left(2 w_{1}+w_{2}+3 w_{3}\right), \\
& 3 \bar{x}_{2}=\sqrt{2 \beta \beta_{1}}\left(2 w_{1}+w_{2}-3 w_{3}\right), \\
& 3 \bar{x}_{3}=2 \sqrt{2 \beta \beta_{1}}\left(w_{1}-w_{2}\right),
\end{aligned}
$$

so that the integration region gets mapped to

$$
\begin{aligned}
& \bar{x}_{1} \in(-\infty, 0] \rightarrow 2 w_{1} \in\left[-w_{2}+3 w_{3},-w_{2}-3 w_{3}\right], \\
& \bar{x}_{2} \in[0,+\infty) \rightarrow w_{2} \in(-\infty,+\infty), \\
& \bar{x}_{3} \in(-\infty,+\infty) \rightarrow w_{3} \in(-\infty, 0] .
\end{aligned}
$$

Applying this transformation we find 


$$
I_{i}=\frac{\sqrt{2}}{3}\left(4 \beta \beta_{1}\right)^{3 / 2} \int_{-\infty}^{0} d w_{3} e^{-\left(\beta_{1}+2 \beta\right) w_{3}^{2}} \int_{-\infty}^{+\infty} d w_{2} e^{-\beta_{1} w_{2}^{2}} \int_{\frac{-w_{2}+3 w_{3}}{2}}^{\frac{-w_{2}-3 w_{3}}{2}} d w_{1} \operatorname{sign}\left(w_{1}-w_{2}\right) \bar{x}_{i}\left(w_{j}\right),
$$

where the prefactor comes from the Jacobian in the change of variables. Solving the integral as written in the $w_{i}$ variables is simpler, but still tedious. The final result in each case is given by

$$
\begin{aligned}
\frac{\beta_{1} I_{1}}{4 \beta_{1} \beta^{2}} & =\frac{\beta_{1}}{\beta_{1}+2 \beta}-\frac{2 \beta_{1} \beta}{\sqrt{\beta_{1}}\left(\beta_{1}+2 \beta\right)^{3 / 2}} \tan ^{-1} \sqrt{\frac{\beta_{1}}{\beta_{1}+2 \beta}}, \\
\frac{\beta I_{2}}{4 \beta_{1} \beta^{2}} & =\frac{\beta}{\beta_{1}+2 \beta}-\frac{2 \beta^{2}}{\sqrt{\beta_{1}}\left(\beta_{1}+2 \beta\right)^{3 / 2}} \tan ^{-1} \sqrt{\frac{\beta_{1}}{\beta_{1}+2 \beta}}, \\
\frac{\beta I_{3}}{4 \beta_{1} \beta^{2}} & =\frac{\beta}{\beta_{1}+2 \beta}+\frac{2 \beta\left(\beta_{1}+\beta\right)}{\sqrt{\beta_{1}}\left(\beta_{1}+2 \beta\right)^{3 / 2}} \tan ^{-1} \sqrt{\frac{\beta_{1}}{\beta_{1}+2 \beta}} .
\end{aligned}
$$

Summing these three terms as indicated in (A8), we get a nice cancellation such that the factor between curly brackets in (A8) is equal to one.

This gives an explicit expression for $G_{0}\left(\beta_{1}, \beta, \beta\right)$. To get the result for arbitrary values of $\beta_{i}$ we note that the symmetry of the observable under arbitrary exchanges $\beta_{i} \leftrightarrow \beta_{j}$ uniquely fixes $\left(\beta_{1}+2 \beta\right) \rightarrow\left(\beta_{1}+\beta_{2}+\beta_{3}\right)$ and $\beta_{1} \beta^{2} \rightarrow \beta_{1} \beta_{2} \beta_{3}$. Putting everything together, we arrive at the final result in Eq. (26).

\section{APPENDIX B: HIGHER GENUS PERTURBATIVE CORRECTIONS}

In this Appendix we compute perturbative $\hbar$ corrections to the one point function $\left\langle\operatorname{Tr} e^{-\beta Q^{2}}\right\rangle$ using (14), by calculating the subleading terms in (22). One way of doing this is by using the expansion for the resolvent of the operator $\mathcal{H}_{s}=-\left(\hbar \partial_{x}\right)^{2}+r(x)^{2}-s \hbar r^{\prime}(x)$, worked out long ago by Gel'fand and Dikii [77] and given by

$$
\left\langle x\left|\frac{1}{\mathcal{H}_{s}-\xi}\right| x\right\rangle=\frac{1}{\hbar} \sum_{p=0}^{\infty} \frac{1}{(-\xi)^{p+1 / 2}} \frac{(2 p-1) ! \tilde{R}_{p}\left[u_{s}(x)\right]}{(-4)^{p} p !(p-1) !},
$$

where $\xi<0$ and we defined $u_{s}(x)=r(x)^{2}-s \hbar r^{\prime}(x)$. The Gel'fand-Dikii functionals $\tilde{R}_{p}\left[r(x)^{2}\right]$ are polynomials in $r(x)^{2}$ and its derivatives computed from the following recursion relation:

$$
\tilde{R}_{p+1}=\frac{2 p+2}{2 p+1}\left[u_{s}(x) \tilde{R}_{p}-\frac{\hbar^{2}}{4} \tilde{R}_{p}^{\prime \prime}-\frac{1}{2} \int^{x} d \bar{x} u_{s}^{\prime}(x) \tilde{R}_{p}\right]
$$

with $\tilde{R}_{0}=1$. Our normalization of the functionals $\tilde{R}_{p}$ is different from the one used in Ref. [77], as we have defined things differently so that $\tilde{R}_{p}=u_{s}(x)^{p}+\mathcal{O}\left(\hbar^{2}\right)$. Explicit expressions for the first few functionals are given in Eq. (10) of Ref. [77]. Applying an inverse Laplace transformation in $\xi$ to Eq. (B1) we obtain the analogous expansion for $\left\langle x\left|e^{-\beta \mathcal{H}_{s}}\right| x\right\rangle$ :

$$
\left\langle x\left|e^{-\beta \mathcal{Q}^{2}}\right| x\right\rangle=\frac{1}{2 \hbar \sqrt{\pi \beta}} \sum_{p=0}^{\infty} \frac{(-\beta)^{p}}{p !} \tilde{R}_{p}\left[u_{s}(x)\right]
$$

While this asymptotic series formula is valid to all orders in $\hbar$, we cannot exchange the infinite series with the $x$ integral in (14). Instead we must solve the series order by order in $\hbar$. To do so, we can use the recursion relation (B2) to expand $\tilde{R}_{p}$ in $\hbar$. The expansion becomes simpler when written in terms of $u(x)=r(x)^{2}$, so that we find

$$
\begin{aligned}
\tilde{R}_{p}\left[u_{s}\right]= & u_{s}^{p}-\frac{\hbar^{2}}{12} p(p-1) u_{s}^{p-3}\left[2 u_{s} u_{s}^{\prime \prime}+(p-2)\left(u_{s}^{\prime}\right)^{2}\right]+\frac{\hbar^{4}}{1440} p(p-1)(p-2) u_{s}^{p-6}\left[24 u_{s}^{3} u_{s}^{(4)}+48(p-3) u_{s}^{2} u_{s}^{\prime} u_{s}^{\prime \prime \prime}\right. \\
& \left.+36(p-3) u_{s}^{2}\left(u_{s}^{\prime \prime}\right)^{2}+44(p-3)(p-4) u_{s}\left(u_{s}^{\prime}\right)^{2} u_{s}^{\prime \prime}+5(p-3)(p-4)(p-5)\left(u_{s}^{\prime}\right)^{4}\right]+\mathcal{O}\left(\hbar^{6}\right) .
\end{aligned}
$$

Inserting this into (B3) and solving the series order by order in $\hbar$ we find 


$$
\begin{aligned}
\left\langle x\left|e^{-\beta \mathcal{H}_{s}}\right| x\right\rangle= & \frac{e^{-\beta u_{s}}}{2 \hbar \sqrt{\pi \beta}}\left[1-\frac{\hbar^{2} \beta^{2}}{12}\left(2 u_{s}^{\prime \prime}-\beta\left(u_{s}^{\prime}\right)^{2}\right)\right. \\
& \left.-\frac{\hbar^{4} \beta^{3}}{1440}\left(24 u_{s}^{(4)}-48 \beta u_{s}^{\prime} u_{s}^{(3)}-36 \beta\left(u_{s}^{\prime \prime}\right)^{2}+44 \beta^{2}\left(u_{s}^{\prime}\right)^{2} u_{s}^{\prime \prime}-5 \beta^{3}\left(u_{s}^{\prime}\right)^{4}\right)+\mathcal{O}\left(\hbar^{6}\right)\right] .
\end{aligned}
$$

The final expansion used in the main text (30) is obtained by replacing $u_{s}(x)=r(x)^{2}-s \hbar r^{\prime}(x)$ with $r(x)$ in (19) and expanding in $\hbar$ one last time.

To check we did not miss any factor in this expansion, let us use it to compute higher $\hbar$ corrections for the toy model defined from $r(x)=\hbar c / x$. Using (14) we find

$$
\begin{aligned}
\left\langle\operatorname{Tr} e^{-\beta Q^{2}}\right\rangle & =\sum_{s= \pm} \frac{1}{2 \hbar \sqrt{\pi \beta}} \int_{0}^{\mu} d x\left[1-\frac{c(c+s) \beta \hbar^{2}}{x^{2}}+\frac{c(c+s)(c(c+s)-2) \beta^{2} \hbar^{4}}{2 x^{4}}+\mathcal{O}\left(\hbar^{6}\right)\right] \\
& =\sum_{s= \pm} \frac{\mu}{2 \hbar \sqrt{\pi \beta}}\left[1+\frac{c(c+s) \beta \hbar^{2}}{\mu^{2}}-\frac{c(c+s)(c(c+s)-2) \beta^{2} \hbar^{4}}{6 \mu^{4}}+\mathcal{O}\left(\hbar^{6}\right)\right],
\end{aligned}
$$

where the integration region is given by $x \in[0, \mu]$ since it is for this range that $r(x)=\hbar c / x$ is well defined. There is a divergent contribution coming from the $x \rightarrow 0$ limit of the integral that we have disregarded. From this expression it is straightforward to compute the spectral density $\rho(q)$ and find

$$
\rho(q)=\sum_{s= \pm} \frac{\mu}{2 \pi \hbar}\left[1-\frac{c(c+s) \hbar^{2}}{2 \mu^{2} q^{2}}-\frac{c(c+s)(c(c+s)-2) \hbar^{4}}{8 \mu^{4} q^{4}}+\mathcal{O}\left(\hbar^{6}\right)\right] .
$$

Comparing this with the first perturbative terms obtained from expanding the exact expression in (37), we find perfect agreement.

\section{APPENDIX C: EIGENFUNCTIONS NORMALIZATION}

In this Appendix we show how to normalize the eigenfunctions $\mathcal{H}_{s} \psi_{\mathcal{E}, s}(x)=\mathcal{E} \psi_{\mathcal{E}, s}(x)$ from their classical $\hbar \rightarrow 0$ behavior. To do so, let us start by writing an expression for $\rho_{0}(q)$ from the first line in (23). After applying an inverse Laplace transform we obtain the following expression for $\rho_{0}(x)$ in terms of $r_{0}(x)$ :

$$
\rho_{0}(q)=\frac{|q|}{2 \pi \hbar} \int_{-\infty}^{\mu} d x \frac{\Theta\left[q^{2}-r_{0}(x)^{2}\right]}{\sqrt{q^{2}-r_{0}(x)^{2}}}
$$

which is precisely the perturbative term obtained from the WKB approximation (42). Comparing with the full expression for $\rho(q)$ in (17), we obtain the following condition satisfied by $\left|\psi_{\mathcal{E}, s}(x)\right|^{2}$ :

$$
\lim _{\hbar \rightarrow 0}\left|\psi_{\mathcal{E}, s}(x)\right|^{2}=\frac{1}{2 \pi \hbar \sqrt{\mathcal{E}}}+\text { oscillating, } \quad x>0
$$

where we have used that $r_{0}(x)=0$ for $x>0$. Since $\psi_{\mathcal{E}, s}(x)$ for $x \rightarrow+\infty$ behaves like a free particle, we expect to have additional oscillating terms which average to zero. Using this condition we can unambiguously fix the normalization constant in the eigenfunctions $\psi_{\mathcal{E}, s}(x)$. Let us see how this works for the toy model eigenfunctions (35). Computing the norm square and taking the classical limit we find

$$
\begin{aligned}
& \lim _{\hbar \rightarrow 0}\left|\psi_{\mathcal{E}, s}(x)\right|^{2} \\
& =\frac{1}{2 \pi \hbar \sqrt{\mathcal{E}}}-\frac{1}{2 \pi \hbar \sqrt{\mathcal{E}}} \cos \left[\frac{2 x \sqrt{\mathcal{E}}}{\hbar}-\frac{\pi}{2}(2 c+s-1)\right],
\end{aligned}
$$

which is precisely the normalization required by Eq. (C2).
[1] R. Jackiw, Nucl. Phys. B252, 343 (1985).

[2] C. Teitelboim, Phys. Lett. 126B, 41 (1983).

[3] A. Achucarro and M. E. Ortiz, Phys. Rev. D 48, 3600 (1993).
[4] A. Fabbri, D. Navarro, and J. Navarro-Salas, Nucl. Phys. B595, 381 (2001).

[5] P. Nayak, A. Shukla, R. M. Soni, S. P. Trivedi, and V. Vishal, J. High Energy Phys. 09 (2018) 048. 
[6] K. S. Kolekar and K. Narayan, Phys. Rev. D 98, 046012 (2018).

[7] A. Ghosh, H. Maxfield, and G. J. Turiaci, J. High Energy Phys. 05 (2020) 104.

[8] S. Sachdev and J. Ye, Phys. Rev. Lett. 70, 3339 (1993).

[9] A. Kitaev, KITP seminars, April 7th and May 27th, University of California, Santa Barbra (UCSB) (2015).

[10] K. Jensen, Phys. Rev. Lett. 117, 111601 (2016).

[11] J. Maldacena and D. Stanford, Phys. Rev. D 94, 106002 (2016).

[12] J. Maldacena, D. Stanford, and Z. Yang, Prog. Theor. Exp. Phys. 2016, 12C104 (2016).

[13] J. Engelsöy, T. G. Mertens, and H. Verlinde, J. High Energy Phys. 07 (2016) 139.

[14] G. Penington, J. High Energy Phys. 09 (2020) 002.

[15] A. Almheiri, N. Engelhardt, D. Marolf, and H. Maxfield, J. High Energy Phys. 12 (2019) 063.

[16] A. Almheiri, T. Hartman, J. Maldacena, E. Shaghoulian, and A. Tajdini, J. High Energy Phys. 05 (2020) 013.

[17] G. Penington, S. H. Shenker, D. Stanford, and Z. Yang, arXiv:1911.11977.

[18] P. Saad, S. H. Shenker, and D. Stanford, arXiv:1903 .11115 .

[19] E. Brezin and V. A. Kazakov, Phys. Lett. B 236, 144 (1990).

[20] M. R. Douglas and S. H. Shenker, Nucl. Phys. B335, 635 (1990).

[21] D. J. Gross and A. A. Migdal, Phys. Rev. Lett. 64, 127 (1990).

[22] D. J. Gross and A. A. Migdal, Nucl. Phys. B340, 333 (1990).

[23] M. Mirzakhani, Inventiones Mathematicae 167, 179 (2006).

[24] B. Eynard, J. High Energy Phys. 11 (2004) 031.

[25] B. Eynard and N. Orantin, Commun. Num. Theor. Phys. 1, 347 (2007).

[26] C. V. Johnson, Phys. Rev. D 101, 106023 (2020).

[27] D. Stanford and E. Witten, Adv. Theor. Math. Phys. 24, 1475 (2020).

[28] F. Dyson, J. Math. Phys. (N.Y.) 3, 140 (1962).

[29] A. Altland and M. R. Zirnbauer, Phys. Rev. B 55, 1142 (1997).

[30] W. Fu, D. Gaiotto, J. Maldacena, and S. Sachdev, Phys. Rev. D 95, 026009 (2017); 95, 069904(A) (2017).

[31] D. Stanford and E. Witten, J. High Energy Phys. 10 (2017) 008.

[32] T. Li, J. Liu, Y. Xin, and Y. Zhou, J. High Energy Phys. 06 (2017) 111.

[33] T. Kanazawa and T. Wettig, J. High Energy Phys. 09 (2017) 050.

[34] F. Sun and J. Ye, Phys. Rev. Lett. 124, 244101 (2020).

[35] S. Forste and I. Golla, Phys. Lett. B 771, 157 (2017).

[36] J. Murugan, D. Stanford, and E. Witten, J. High Energy Phys. 08 (2017) 146.

[37] C. V. Johnson, Phys. Rev. D 103, 046012 (2021).

[38] C. V. Johnson, Phys. Rev. D 103, 046013 (2021).

[39] C. V. Johnson, arXiv:2008.13120.

[40] T. R. Morris, Nucl. Phys. B356, 703 (1991).

[41] S. Dalley, C. V. Johnson, and T. Morris, Nucl. Phys. B368, 625 (1992).
[42] S. Dalley, C. Johnson, T. Morris, and A. Watterstam, Mod. Phys. Lett. A 07, 2753 (1992).

[43] S. Dalley, C. V. Johnson, and T. Morris, Nucl. Phys. B368, 655 (1992).

[44] S. Dalley, C. V. Johnson, and T. Morris, Nucl. Phys. B, Proc. Suppl. 25A, 87 (1992).

[45] I. R. Klebanov, J. M. Maldacena, and N. Seiberg, Commun. Math. Phys. 252, 275 (2004).

[46] T. G. Mertens and G. J. Turiaci, J. High Energy Phys. 01 (2021) 073.

[47] T. G. Mertens and G. J. Turiaci, J. High Energy Phys. 08 (2019) 127.

[48] G. J. Turiaci, M. Usatyuk, and W. W. Weng, arXiv:2011 .06038 .

[49] T. G. Mertens, J. High Energy Phys. 04 (2021) 245.

[50] P. Betzios and O. Papadoulaki, J. High Energy Phys. 09 (2020) 125.

[51] J. Polchinski String Theory Volume 2: Superstring Theory and Beyond (Cambridge University Press, Cambridge, England, 1998), p. 531.

[52] P. H. Ginsparg and G. W. Moore, in Theoretical Advanced Study Institute (TASI 92): From Black Holes and Strings to Particles (1993), pp. 277-469.

[53] V. Periwal and D. Shevitz, Phys. Rev. Lett. 64, 1326 (1990).

[54] V. Periwal and D. Shevitz, Nucl. Phys. B344, 731 (1990).

[55] C. R. Nappi, Mod. Phys. Lett. A 05, 2773 (1990).

[56] C. Crnkovic and G. W. Moore, Phys. Lett. B 257, 322 (1991).

[57] T. J. Hollowood, L. Miramontes, A. Pasquinucci, and C. Nappi, Nucl. Phys. B373, 247 (1992).

[58] C. Crnkovic, M. R. Douglas, and G. W. Moore, Int. J. Mod. Phys. A 07, 7693 (1992).

[59] M. R. Douglas, N. Seiberg, and S. H. Shenker, Phys. Lett. B 244, 381 (1990).

[60] D. J. Gross and E. Witten, Phys. Rev. D 21, 446 (1980).

[61] G. M. Cicuta, L. Molinari, and E. Montaldi, Mod. Phys. Lett. A 01, 125 (1986).

[62] G. Akemann, J. Baik, and P. Di Francesco, The Oxford Handbook of Random Matrix Theory, Oxford Handbooks in Mathematics (Oxford University Press, New York, 2011).

[63] P. Di Francesco, P. H. Ginsparg, and J. Zinn-Justin, Phys. Rep. 254, 1 (1995).

[64] V. A. Kazakov, Mod. Phys. Lett. A 04, 2125 (1989).

[65] B. Eynard, T. Kimura, and S. Ribault, arXiv:1510.04430.

[66] D. Anninos and B. Mühlmann, J. Stat. Mech. (2020) 083109.

[67] H. Neuberger, Nucl. Phys. B352, 689 (1991).

[68] E. Brezin, C. Itzykson, G. Parisi, and J. B. Zuber, Commun. Math. Phys. 59, 35 (1978).

[69] D. Bessis, C. Itzykson, and J. B. Zuber, Adv. Appl. Math. 1, 109 (1980).

[70] V. Zakharov and A. Shabat, Funkts. Anal. Prilozhen. 8, 54 (1974).

[71] To be published.

[72] T. Banks, M. R. Douglas, N. Seiberg, and S. H. Shenker, Phys. Lett. B 238, 279 (1990).

[73] C. V. Johnson and F. Rosso, J. High Energy Phys. 04 (2021) 030. 
[74] D. J. Gross and A. A. Migdal, Nucl. Phys. B340, 333 (1990).

[75] J. Ambjorn, J. Jurkiewicz, and Y. Makeenko, Phys. Lett. B 251, 517 (1990).

[76] G. W. Moore, N. Seiberg, and M. Staudacher, Nucl. Phys. B362, 665 (1991).

[77] I. Gelfand and L. Dikii, Russ. Math. Surv. 30, 77 (1975).

[78] T. Banks, M. R. Douglas, N. Seiberg, and S. H. Shenker, Phys. Lett. B 238, 279 (1990).

[79] P. Forrester, Log-Gases and Random Matrices, London Mathematical Society Monographs (LMS-34) (Princeton University Press, Princeton, NJ, 2010).

[80] G. E. Andrews, R. Askey, and R. Roy, Special Functions, Encyclopedia of Mathematics and its Applications (Cambridge University Press, Cambridge, England, 1999).

[81] J. S. Cotler, G. Gur-Ari, M. Hanada, J. Polchinski, P. Saad, S. H. Shenker, D. Stanford, A. Streicher, and M. Tezuka, J. High Energy Phys. 05 (2017) 118; 09 (2018) 002(E).

[82] T. Guhr, A. Muller-Groeling, and H. A. Weidenmuller, Phys. Rep. 299, 189 (1998).

[83] J. Liu, Phys. Rev. D 98, 086026 (2018).

[84] R. M. Miura, J. Math. Phys. (N.Y.) 9, 1202 (1968).

[85] R. M. Miura, C. S. Gardner, and M. D. Kruskal, J. Math. Phys. (N.Y.) 9, 1204 (1968).

[86] T. R. Morris, Fermilab, Report No. FERMILAB-PUB-90136-T.

[87] S. Mizoguchi, Nucl. Phys. B716, 462 (2005).

[88] K. Okuyama and K. Sakai, J. High Energy Phys. 10 (2020) 160.

[89] V. G. Drinfeld and V. V. Sokolov, J. Sov. Math. 30, 1975 (1985).
[90] E. Date, M. Jimbo, M. Kashiwara, and T. Miwa, Publ. Res. Inst. Math. Sci. Kyoto 18, 1077 (1982).

[91] M. Jimbo and T. Miwa, Publ. Res. Inst. Math. Sci. Kyoto 19, 943 (1983).

[92] G. Segal and G. Wilson, Inst. Hautes Etudes Sci. Publ. Math. 61, 5 (1985).

[93] V. G. Kac and M. Wakimoto, Proc. Symp. Pure Math. 49, 191 (1989).

[94] T. J. Hollowood, L. Miramontes, A. Pasquinucci, and C. Nappi, Nucl. Phys. B373, 247 (1992).

[95] G. R. Harris and E. J. Martinec, Phys. Lett. B 245, 384 (1990).

[96] E. Brezin and H. Neuberger, Phys. Rev. Lett. 65, 2098 (1990).

[97] E. Brezin and H. Neuberger, Nucl. Phys. B350, 513 (1991).

[98] B. A. Kupershmidt, Commun. Math. Phys. 99, 51 (1985).

[99] R. Iyer, C. V. Johnson, and J. S. Pennington, J. Phys. A 44, 015403 (2011).

[100] R. Iyer, C. V. Johnson, and J. S. Pennington, J. Phys. A 44, 375401 (2011).

[101] H. Maxfield and G. J. Turiaci, J. High Energy Phys. 01 (2021) 118.

[102] E. Witten, Proc. R. Soc. A 476, 20200582 (2020).

[103] J. Maldacena, G. J. Turiaci, and Z. Yang, J. High Energy Phys. 01 (2021) 139.

[104] J. Cotler, K. Jensen, and A. Maloney, J. High Energy Phys. 06 (2020) 048.

[105] K. Okuyama, J. High Energy Phys. 10 (2018) 037.

[106] K. Okuyama and K. Sakai, J. High Energy Phys. 08 (2020) 126. 